\title{
Making precise predictions of the Casimir force between metallic plates via a weighted Kramers-Kronig transform
}

\author{
Giuseppe Bimonte* \\ Dipartimento di Scienze Fisiche Università di Napoli Federico II Complesso Universitario MSA, \\ Via Cintia I-80126 Napoli Italy and INFN Sezione di Napoli, ITALY
}

(Dated: August 25, 2018)

\begin{abstract}
The possibility of making precise predictions for the Casimir force is essential for the theoretical interpretation of current precision experiments on the thermal Casimir effect with metallic plates, especially for sub-micron separations. For this purpose it is necessary to estimate very accurately the dielectric function of a conductor along the imaginary frequency axis. This task is complicated in the case of ohmic conductors, because optical data do not usually extend to sufficiently low frequencies to permit an accurate evaluation of the standard Kramers-Kronig integral used to compute $\epsilon(i \xi)$. By making important improvements in the results of a previous paper by the author, it is shown that this difficulty can be resolved by considering suitable weighted dispersions relations, which strongly suppress the contribution of low frequencies. The weighted dispersion formulae presented in this paper permit to estimate accurately the dielectric function of ohmic conductors for imaginary frequencies, on the basis of optical data extending from the IR to the UV, with no need of uncontrolled data extrapolations towards zero frequency that are instead necessary with standard Kramers-Kronig relations. Applications to several sets of data for gold films are presented to demonstrate viability of the new dispersion formulae.
\end{abstract}

PACS numbers: 05.30.-d, 77.22.Ch, 12.20.Ds

Keywords: Casimir, dispersion relations.

\section{INTRODUCTION}

The Casimir effect, and more in general dispersion forces, are the current object of much theoretical and experimental interest. The reasons for the continuing interest in this well-established field are numerous, and are connected with both fundamental and applied science. For an updated overview of the subject, the reader is referred to recent reviews $[1-3]$.

After fifty years of slow progress, the field of Casimir physics received a strong push in the last decade, as a result of a new wave of experiments [4] which succeeded for the first time in measuring the tiny Casimir force with unprecedented precision. The new measurements provided a definitive confirmation of this phenomenon, but at the same time experiments utilizing metallic surfaces (which are used in the majority of current experiments) have raised unexpected theoretical puzzles, for the so-called thermal Casimir force. The problem is whether in the computation of the Casimir force at finite temperature conduction electrons in the plates should be described as a collisionless plasma, similarly to what is done in infrared optics, or if relaxation processes should be included [2, 5- 7]. The two approaches have been dubbed in the Casimir literature as plasma and Drude prescriptions, respectively. The problem is rather subtle indeed, with non trivial implications in thermodynamics and statistical physics that are not yet fully understood. In fact,

*Bimonte@na.infn.it\% it has been found that inclusion of relaxation effects in the idealized case of two plates with no imperfections leads to violation of the Nernst theorem [8], while no such violation occurs when any amount of impurities is present [9]. It has been shown however that neglect of relaxation processes leads to contradiction with the Bohrvan Leeuwen theorem of classical statistical physics [10]. From an experimental point of view, the resolution of the problem requires observation of the thermal Casimir force between two metallic plates, because the two alternative theoretical pictures give different predictions for its magnitude. The experimental situation is perplexing, and at the moment of this writing there appears to be a striking contradiction between the results of different experiments by different groups. A series of experiments using microtorsional oscillators by the Purdue group [11], providing at this time the most precise measurements of the Casimir force between metallic bodies in the separation range from $160 \mathrm{~nm}$ to $750 \mathrm{~nm}$, were shown to be in agreement with the plasma prescription and to rule out the Drude prescription. Also a large distance torsion balance experiment [12] in which the Casimir force was measured in the separation range from 0.48 to $6.5 \mu \mathrm{m}$, obtained results that are in agreement with the assumption of ideal metal plates, and are in contradiction with the Drude model. On the contrary, a new experiment 13] in which the Casimir force between a spherical lens and a flat plate was measured in the range from $700 \mathrm{~nm}$ to $7 \mu \mathrm{m}$ by a torsional balance, was claimed to be fully consistent with the Drude prescription, and to rule out the plasma one. This state of things calls for a careful investigation, to see if the contradiction can be resolved.

It is well known by now [2] that the theoretical in- 
terpretation of precision Casimir experiments is a difficult problem, because several possible sources of error must be considered, that include for example consideration of unavoidable imperfections of the surfaces, like roughness and other uncontrolled geometric distortions [14], the possible presence of residual electrostatic forces etc. We shall not be concerned with these issues here, and for a thorough discussion of the problem of theoryexperiment comparison in Casimir experiments, we address the reader to the recent work [15, 16]. In this paper we shall focuss our attention on the influence of the optical properties of the the plates, described by their dielectric function $\epsilon(\omega)$, which by far constitutes the dominant factor affecting the magnitude of the Casimir force, especially at sub-micron separations. The effect of the optical properties of the plates on the Casimir force must be estimated very precisely, if one seeks to discriminate between alternative prescriptions for the thermal Casimir effect, because the predicted magnitudes of the force resulting from different approaches differ by just a few per cent at separations below one micron, which is the range investigated in the experiments by the Purdue group.

According to the theory of dispersion forces [1] in order to compute the Casimir force between two dielectric (non-magnetic) plates of any shape at temperature $T$ one needs to know the electric permittivities $\epsilon\left(i \xi_{n}\right)$ of the materials constituting the plates, evaluated at certain temperature-dependent discrete imaginary frequencies $\xi_{n}=2 \pi n k_{B} T / \hbar$, where $n$ is a non-negative integer, known as Matsubara frequencies. Getting accurate values for the dielectric function $\epsilon(i \xi)$ is not easy, in particular when ohmic conductors are considered. This is so because the imaginary-frequency dielectric function $\epsilon(i \xi)$ cannot be directly measured by any experiment, and therefore it has to be computed using Kramers-Kronig (KK) dispersion relations, starting from optical data of the involved materials. We shall specifically consider the case of gold, which is the material of interest in the recent experiments probing the thermal effect. Until a few years ago [17 19] it was customary to compute $\epsilon(i \xi)$ by using the data for gold tabulated in the handbook [20]. However, in the most recent experiments the new practice has been adopted of computing the quantities $\epsilon\left(i \xi_{n}\right)$ on the basis of optical data measured directly on the samples used in the Casimir measurements. The necessity of doing this was first stressed in [21] and it stems from the fact that optical properties of thin polycrystalline gold films, typically used in Casimir experiments, are much dependent on the used deposition method. It has been claimed [22, 23] that possible sample-to-sample variations of the optical properties may engender significant variations in the Casimir force, possibly larger than 10 per cent at submicron separations. While the large differences in the Casimir force reported in [22] probably do not arise for carefully prepared gold films like those used in the experiments [11], it seems correct to state that the optical properties of the used samples should be carefully characterized, if per cent precision in the theory-experiment comparison is aimed at.

A big practical problem remains however because, due to the characteristic $1 / \omega$ singularity displayed by the dielectric function of ohmic conductors, the standard KK integral used to compute $\epsilon(i \xi)$ (Eq. (1) below) receives a very large contribution from frequencies below the IR, for which it is very difficult to collect optical data for thin metallic films. The remedy to this problem, initially adopted in [24] and followed afterwards by all authors, consists in extrapolating the available data below the minimum accessible frequency $\omega_{\min }$, in order to estimate the KK integral in the interval $0<\omega<\omega_{\text {min }}$ where optical data are not directly available. The extrapolation is usually done by means of the simple Drude formula for the dielectric function of ohmic conductors. It is important to observe that this contribution is usually very large (it easily accounts for more than fifty per cent of the KK integral) and therefore the values of the Casimir force that are obtained by this procedure are very sensitive to experimental uncertainties in the values of the Drude parameters. For example, in Ref. [22] it was estimated that experimental uncertainties in the Drude parameters may imply by themselves an uncertainty in the Casimir force as large as $1 \%$ at $100 \mathrm{~nm}$.

In our opinion, the reliability of the whole procedure described above can be questioned, because in reality there is no way to know how accurately the Drude model describes the dielectric function of gold films, in the wide range of frequencies for which it is used in Casimir computations. It seems to us that there is no way to quantify the error determined by possible deviations of the actual dielectric function from the Drude model. In our judgment, in order to obtain fully reliable predictions of the Casimir force with metallic plates it is necessary to find means of reducing the impact of the Drude extrapolation. In principle the straightforward way to achieve this goal would be by further extending optical data on the low frequency side. It is unlikely however that much progress can be made in this way, since it has been estimated that a determination of the Casimir force between two gold plates with an error of half per cent at a separation of $150 \mathrm{~nm}$ solely on the basis of measured optical data, would require extending optical measurements till wavelengths in the millimeter region, which is a very difficult thing to do.

An alternative approach, which does not require further extension of optical measurements beyond the limits of currently available optical data, was proposed recently by the author in Ref. [25]. The idea is to introduce in the dispersion relations used to compute $\epsilon(i \xi)$ suitable analytical weights $f(z)$, called by us "window" functions, that suppress the contribution of frequencies for which no optical data are available. We should observe that consideration of weighted dispersion relations is well documented in the optical literature, where they have been exploited for example to derive new important relations connecting the refraction index to the extinction coefficient. For details, we address the reader to Chapter 3 
of [20]. In our first work [25] we demonstrated that by taking weights $f(z)$ that vanish both at zero and at infinity, it is indeed possible to suppress very effectively the contribution of frequencies outside the data interval $\omega_{\min }<\omega<\omega_{\max }$. A simple family of window functions, parametrized by two integers $p$ and $q$ (with $p<q$ ) and by a complex frequency $w$, was offered there, and it was shown that for suitable choices of the parameters the error made by truncating the integral to the frequency range $\omega_{\min }<\omega<\omega_{\max }$ can be made negligible at both ends of the integration domain, in such a way that a precise determination of $\epsilon(i \xi)$ is possible, without extrapolating the optical data outside the interval where they are available. The key feature of the generalized dispersion relation that makes this possible is that, differently from the standard KK relation which only involves $\epsilon^{\prime \prime}(\omega)$, the generalized relations involve both $\epsilon^{\prime}(\omega)$ and $\epsilon^{\prime \prime}(\omega)$, and therefore they exploit the full information delivered by optical data. This is not a problem in principle if one considers that optical techniques exist, like ellipsometry, that permit to measure independently both $\epsilon^{\prime}(\omega)$ and $\epsilon^{\prime \prime}(\omega)$ with good precision. Such a technique was used for example in [22].

The analysis in [25] was unrealistic however, in that it assumed that optical data in the interval $\omega_{\min }<\omega<$ $\omega_{\max }$ were known with an infinite precision. Real optical data of course are not like that, as they are affected by experimental errors of various nature. That the analysis in 25] was oversimplified has been pointed out recently in [15], where the windowed dispersion relations were tentatively applied to the tabulated data for gold of Ref. [20]. Using the same window functions and the same window parameters used in [25], the authors of [15] obtained negative values for the quantity $\epsilon(i \xi)$ in certain intervals of the imaginary axis, and this is absolutely unacceptable because on general grounds one knows that along the imaginary axis the electric permittivity of a causal medium must be positive [26]. In an attempt at understanding their findings, the authors of Ref. [15] observed that the handbook [20] combines data from several distinct experiments, using gold films prepared by different procedures. Since, as we said earlier, optical properties of gold films depend significantly on the deposition method, the operation of combining optical data for films having exceedingly different properties (that this is indeed the case with the tabulated data is shown in Sec. V below) may in principle spoil the overall KK consistency of the data, which is an essential precondition for dispersion relations to work well. We agree with the authors of Ref. [15], though, that this potential problem with the tabulated data of Ref. 20] cannot explain the obtained negative values of $\epsilon(i \xi)$. They argue that the main reason is the possible strong sensitivity of the windowed dispersion relations to experimental errors in the optical data, in particular in the real part of the permittivity. If an exceedingly large amplification of small uncertainties in the optical data were an unavoidable feature of our windowed dispersion relations, their practical utility would be severely diminished, of course. The issue of error propagation is an important point deserving a detailed analysis, and we address it in the present paper. We have performed a Monte Carlo simulation to determine how random errors in the optical data are propagated by the windowed dispersion relations. The results of these simulation confirm the suspicion of the authors of Ref. [15], and explain their findings, revealing that the choice of the window functions that was made in 25], was very unfortunate indeed, because it leads to a huge amplification of errors in the optical data. Trying to resolve the problem, we realized that the key property protecting the standard KK relation from this sort of instability is the fact that it involves a positive definite kernel. This property was badly violated by the weighted dispersions relations considered in [25], which are instead characterized by kernels attaining large negative and positive values. After several attempts, we could eventually fix this problem and we found a new class of weight functions, presented in this paper, that lead to positive kernels when applied to ohmic conductors. In this paper we demonstrate that the new weighted dispersion formulae do not suffer from large error propagation, and therefore they can be used to reliably compute $\epsilon(i \xi)$, and in turn the Casimir force, solely on the basis of optical data extending from the IR to the UV, with no need of further extending optical measurements to lower frequencies.

The plan of the paper is as follows: in Sec. II we review the KK formula routinely used to compute the dielectric function $\epsilon(i \xi)$, and we discuss its weaknesses in the case of ohmic conductors. In Section III, we review briefly the weighted or "windowed" dispersion relations that were introduced in 25], and we present a new class of weights or "window functions" leading to dispersion relations with positive kernels when applied to conductors. In Sec. IV we present our numerical computations which demonstrate how the window functions considered in our first work suffer from a strong instability, and we show that the new weights resolve this problem. In Sec. V we apply the windowed dispersion relations to tabulated optical data for gold of Ref. [20], and in Sec. VI to some of the data in [22]. In Sec. VII we use the weighed dispersion relations to derive an alternative expression for the so-called generalized plasma model that has been recently advocated as providing the correct description for ohmic conductors in the Casimir effect. Finally, Sec VIII contains our conclusions and a discussion of the results.

\section{DIELECTRIC FUNCTION AT IMAGINARY FREQUENCIES}

As it is well known from the theory of dispersion forces [1] the Casimir interaction between two macroscopic bodies at temperature $T$ depends on the dielectric functions of the two bodies, evaluated for a discrete set of imaginary Matsubara frequencies $\xi_{n}=2 \pi n k T / \hbar$, where $n$ is any non-negative integer. Finding means of accurately 
computing the quantities $\epsilon\left(i \xi_{n}\right)$ embodying the material dependence of the Casimir force, is the primary goal of this work. We shall see below that this is not a trivial task, in particular when ohmic conductors are considered. As we said earlier, it is impossible to directly measure $\epsilon(i \xi)$ because any conceivable optical experiment can only determine the dielectric permittivity $\epsilon(\omega)$ along the real frequency axis. Knowledge of optical data along the real axis can be exploited to compute $\epsilon(i \xi)$ by different mathematical procedures, depending on the sought accuracy. For moderate precisions, at five per cent level or so, the simplest method is to make oscillator models of the optical data $\epsilon(\omega)$ for the material of interest. The explicit analyticity of such analytical models then permits to obtain the quantities $\epsilon\left(i \xi_{n}\right)$ by direct substitution $\omega \rightarrow i \xi_{n}$ into the oscillator formula. However, this straightforward procedure is not accurate enough for the purpose of interpreting the most recent precision Casimir experiments. When higher precision is needed, the quantities $\epsilon\left(i \xi_{n}\right)$ must be determined directly on the basis of optical data, without making recourse to simplified analytical models for the latter. This can be done by exploiting dispersion relations entailed by causality of the electric permittivity, which relate the unmeasurable quantities $\epsilon\left(i \xi_{n}\right)$ to the measurable real-frequency permittivity $\epsilon(\omega)$. The dispersion relation that has been utilized until now in the literature is the following $\mathrm{KK}$ relation

$$
\epsilon(i \xi)-1=\frac{2}{\pi} \int_{0}^{\infty} d \omega \frac{\omega \epsilon^{\prime \prime}(\omega)}{\omega^{2}+\xi^{2}},
$$

which is valid for all materials, like insulators and ohmic conductors, whose electric permittivity diverges at most like $1 / \omega$ at zero frequency.

As we explained in the Introduction application of the above formula to ohmic conductors meets with difficulties. We recall first that the important range of Matsubara frequencies that need be considered in Casimir computations depends on the separation $a$ between the two bodies. In practice [2], for an accurate determination of the Casimir force it is sufficient to consider Matsubara frequencies up to a maximum value of about ten times the characteristic frequency $\omega_{c}=2 /(2 a)$. For separations $a$ larger than $50 \mathrm{~nm}$, which are the ones probed by current experiments, is is therefore sufficient to consider Matsubara modes with frequencies $\xi$ ranging from 0.16 $\mathrm{eV} / \hbar$, representing the frequency of the first Matsubara mode at room temperature, up to a maximum frequency of ten $\mathrm{eV} / \hbar$ or so. Now we see from Eq. (1) that in order to evaluate $\epsilon(i \xi)$ at any imaginary frequency $\xi$ it is in principle necessary to know $\epsilon^{\prime \prime}(\omega)$ at all frequencies $\omega$. Unfortunately such a complete knowledge of $\epsilon^{\prime \prime}(\omega)$ is never possible, because optical data are always restricted to some finite frequency range $\omega_{\min }<\omega<\omega_{\max }$, starting from a non-zero minimum frequency $\omega_{\min }>0$. Consider for example the data for gold collected in the handbook [20], which have been routinely utilized to interpret Casimir experiments using gold plates, including the recent experiments [11]. These data, which we shall describe more accurately later on, cover a range of frequencies extending from $0.125 \mathrm{eV} / \hbar$, corresponding to the IR, till $10^{4} \mathrm{eV} / \hbar$. Unfortunately, this range is not sufficiently wide to permit an accurate estimate of the integral on the r.h.s. of Eq. (1) in the relevant range of imaginary frequencies $\xi$, which as we said extends from $0.16 \mathrm{eV} / \hbar$ to ten $\mathrm{eV} / \hbar$. In fact there is no difficulty on the high frequency side, because fall-off properties of $\epsilon^{\prime \prime}(\omega)$ entail that for all $\xi$ 's in this range, real frequencies $\omega$ larger than a few tens of $\mathrm{eV} / \hbar$ give already a negligible contribution to the integral on the r.h.s. of Eq. (1). On the low-frequency side, however, we have a real problem originating from the $1 / \omega$ singularity displayed by the imaginary part of the permittivity of ohmic conductors. As a result $\epsilon^{\prime \prime}(\omega)$ becomes extremely large at low frequencies, in such a way that the integral in Eq. (11) receives a very large contribution from low frequencies for which no optical data are available. Since truncation of the integral to the frequency $\omega_{\text {min }}$ results in a large error, one is forced to extrapolate the dielectric function $\epsilon^{\prime \prime}(\omega)$ to frequencies $\omega<\omega_{\min }$, where optical data are not available, to evaluate the integral for $\omega<\omega_{\min }$. As a rule, the extrapolation is done using the simple Drude model

$$
\epsilon_{\operatorname{Dr}}(\omega)=1-\frac{\omega_{p}^{2}}{\omega(\omega+i \gamma)},
$$

where $\omega_{p}$ is the plasma frequency, and $\gamma$ is the relaxation frequency. Typical values for these parameters for gold are $\omega_{p}=9 \mathrm{eV} / \hbar$ and $\gamma=35 \mathrm{meV} / \hbar$ [24]. As it is well known, the Drude model is expected to provide a reasonable approximation to the permittivity of ohmic conductors for low frequencies, and the hope is that the error resulting from use of the Drude extrapolation is not too large. According to this procedure, the quantity $\epsilon(i \xi)$ in Eq. (11) is computed as

$$
\epsilon(i \xi)=1+\epsilon_{\mathrm{cut}}(i \xi)+\epsilon_{\mathrm{expt}}(i \xi),
$$

where $\epsilon_{\text {cut }}(i \xi)$ is calculated using the Drude extrapolation for $\epsilon(\omega)$ in the unaccessible frequency range $\omega<\omega_{\min }$, while $\epsilon_{\text {expt }}(i \xi)$ is calculated using the experimental optical data $\epsilon_{\text {expt }}^{\prime \prime}(\omega)$, according to the following formulas:

$$
\epsilon_{\mathrm{cut}}(i \xi)=\frac{2}{\pi} \int_{0}^{\omega_{\min }} d \omega \frac{\omega \epsilon_{\mathrm{Dr}}^{\prime \prime}(\omega)}{\omega^{2}+\xi^{2}}
$$

and

$$
\epsilon_{\operatorname{expt}}(i \xi)=\frac{2}{\pi} \int_{\omega_{\min }}^{\infty} d \omega \frac{\omega \epsilon_{\mathrm{expt}}^{\prime \prime}(\omega)}{\omega^{2}+\xi^{2}}
$$

Later on, we shall denote by a subscript KK estimates for the permittivities and Casimir forces obtained by using Eqs. (345). The large weight of the Drude term in Eq. (3) has been clearly recognized recently [22]. Using the handbook data, it has been estimated (see Fig. 10 of [22] and Fig. 4 below) that in the interval of imaginary frequencies $\xi$ from 0.1 to $10 \mathrm{eV} / \hbar$ the quantity $\epsilon_{\text {cut }}(i \xi)$ gives the dominant contribution to $\epsilon(i \xi)$ for $\xi<4 \mathrm{eV} / \hbar$, 
representing over $90 \%$ of the total magnitude of $\epsilon(i \xi)$ for $\xi=0.1 \mathrm{eV} / \hbar$. We remark that the situation does not improve too much if the data interval is extended to longer wavelengths. For example, the authors of Ref. [22] measured by ellipsometry the dielectric functions of several gold films for wavelengths from 33 to $0.14 \mu \mathrm{m}$, corresponding to the frequency interval from about 35 $\mathrm{meV} / \hbar$ to $10 \mathrm{meV} / \hbar$. Despite the fact that IR measurements were extended till photon energies that are roughly one fourth smaller than the handbook data, still $\epsilon_{\text {cut }}(i \xi)$ provides a large fraction of $\epsilon(i \xi)$, about $60 \%$ for $\xi=0.1$ $\mathrm{eV} / \hbar$ and about $40 \%$ for $\xi=1 \mathrm{eV} / \hbar$ (see thick solid line of Fig. 4).

To give a further sense of the importance of the Drude extrapolation for the Casimir effect, we have estimated the error which is made in the Casimir pressure $P(a, T)$ between two gold plates if one neglects altogether the contribution of the Drude extrapolation in Eq. (3). As it is well known, the Casimir force per unit area $P(a, T)$ between two identical plane-parallel homogeneous and isotropic plates, placed in vacuum at a distance $a$ is provided by the following Lifshitz formula [27] (a minus sign corresponds to an attraction between the plates):

$P(a, T)=-\frac{k_{B} T}{\pi} \sum_{n \geq 0}^{\prime} \sum_{\alpha} \int d k_{\perp} k_{\perp} q_{n}\left(\frac{e^{2 a q_{n}}}{r_{\alpha}^{2}\left(i \xi_{n}, k_{\perp}\right)}-1\right)^{-1}$

where the prime over the $n$-sum means that the $n=0$ term has to be taken with a weight one half, $\alpha=\mathrm{TE}, \mathrm{TM}$ is the polarization, $k_{\perp}$ denotes the magnitude of the projection of the wave-vector onto the plane of the plates and $q_{n}=\sqrt{k_{\perp}^{2}+\xi_{n}^{2} / c^{2}}$, where $\xi_{n}=2 \pi n k_{B} T / \hbar$ are the Matsubara frequencies. The quantities $r_{\alpha}\left(i \xi_{n}, k_{\perp}\right)$ denote the familiar Fresnel reflection coefficients of the slabs for $\alpha$-polarization, evaluated at imaginary frequencies $i \xi_{n}$. They have the following expressions:

$$
\begin{gathered}
r_{\mathrm{TE}}\left(i \xi_{n}, k_{\perp}\right)=\frac{q_{n}-k_{n}}{q_{n}+k_{n}}, \\
r_{\mathrm{TM}}\left(i \xi_{n}, \mathbf{k}_{\perp}\right)=\frac{\epsilon\left(i \xi_{n}\right) q_{n}-k_{n}}{\epsilon\left(i \xi_{n}\right) q_{n}+k_{n}},
\end{gathered}
$$

where $k_{n}=\sqrt{k_{\perp}^{2}+\epsilon\left(i \xi_{n}\right) \xi_{n}^{2} / c^{2}}$. Unless explicitly stated otherwise, it is understood that all Casimir pressures here and below are computed using the so-called Drude prescription [2] for ohmic conductors. In this scheme, the $n=0$ mode for TE polarization contributes nothing, and the $n>0$ terms are evaluated using the dielectric function $\epsilon(i \xi)$ of the considered metal, without any modification aimed at suppressing the effect of ohmic dissipation for conduction electrons. In Fig. 1 we plot the fractional difference, in per cent, between the magnitudes $|P(a, T)|$ and $\left|P_{\text {cut }}(a, T)\right|$ of the Casimir pressure between two parallel gold plates at room temperature, that are obtained respectively if in $\epsilon(i \xi)$ one includes or neglects the contribution of the Drude extrapolation $\epsilon_{\mathrm{cut}}(i \xi)$ in Eq. (3).

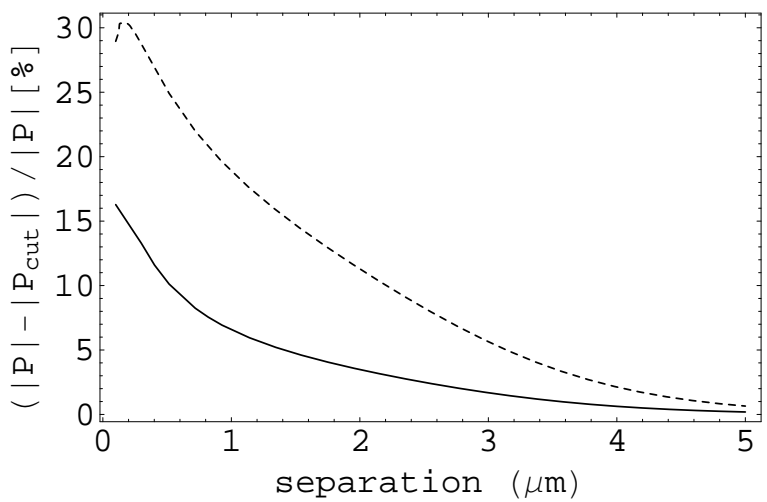

FIG. 1: Per cent error in the theoretical magnitudes of the Casimir pressure between two gold parallel plates at room temperature, resulting from neglect of the low-frequency contribution $\epsilon_{\text {cut }}(i \xi)$ in Eq. (3). The dashed line was computed using the handbook data of Ref.[20] $\left(\omega_{\min }=0.125 \mathrm{eV} / \hbar\right)$. The solid line uses the optical data for sample 5 of Ref. 22] $\left(\omega_{\min }=0.042 \mathrm{eV} / \hbar\right)$.

The dotted curve displayed in the Figure is relative to the handbook data [20], for which $\omega_{\min }=0.125 \mathrm{eV} / \hbar$. Extrapolation to low frequencies was done using the com'monly used values for the Drude parameters quoted in [24], namely $\omega_{p}=9 \mathrm{eV} / \hbar$ and $\gamma=35 \mathrm{meV} / \hbar$. The solid curve in Fig. 1 was instead computed using the optical data relative to sample 5 of Ref. [22], consisting of an annealed gold film deposited on a mica substrate, with a thickness of $120 \mathrm{~nm}$. We have selected this sample, because of the five samples studied in Ref. [22] this one has the closest plasma frequency $\left(\omega_{p}=8.38 \mathrm{eV} / \hbar\right)$ to the commonly accepted value for gold. In 22] the optical data for this film were measured in the frequency interval extending from $\omega_{\min }=0.042 \mathrm{eV} / \hbar$ to $\omega_{\max }=9$ $\mathrm{eV} / \hbar$. The Drude parameters for this film were estimated in [22] to have the values $\omega_{p}=(8.38 \pm 0.08) \mathrm{eV} / \hbar$ and $\gamma=(37.1 \pm 1.9) \mathrm{meV} / \hbar$. When considering this film, the handbook data from [20] were used to extend the data of 22] above $9 \mathrm{eV} / \hbar$. We see from Fig. 11 that at all separations the Drude extrapolation has a smaller weight when the data of [22] are used. This is a result of the fact, noticed already, that optical data of 22] extend to lower frequencies than the tabulated data. We see from the Figure that the influence of the Drude extrapolation on the predicted pressure is very significant for both the handbook data and for the data of [22], especially at submicron separations. For a separation $a$ of $100 \mathrm{~nm}$ it contributes about thirty per cent of the total pressure in the case of the handbook data, and more than fifteen per cent for the data of Ref. [22].

In the recent literature [11, 15] it has been claimed that relying on the handbook data of [20] and by following the procedure outlined above, the magnitude of the Casimir pressure between two parallel gold plates can be predicted theoretically with an uncertainty of $0.5 \%$ in the separation range from $150 \mathrm{~nm}$ to $400 \mathrm{~nm}$. This 
claim does not seem warranted to us, because of the large weight of the Drude extrapolation in the computation of the Casimir force. Considering the magnitude of this contribution, a precision of half per cent at separations around $200 \mathrm{~nm}$ can be claimed only if one is confident that the Drude extrapolation can be used to estimate the contribution to the Casimir pressure of unaccessible low frequencies $\omega<\omega_{\min }$ with a fractional precision better than one or two per cent, a non trivial statement indeed. In our opinion, the reliability of Casimir computations would greatly increase if we could find means of substantially reducing the weight of the Drude extrapolation. We discussed earlier that one cannot expect to achieve this goal by further extending to lower frequencies the data interval. We shall demonstrate below that this goal can be achieved instead by using alternative forms of dispersion relations, which strongly suppress the weight of the Drude extrapolation in determining the dielectric function $\epsilon(i \xi)$.

\section{WEIGHTED KRAMERS-KRONIG RELATIONS}

In the work 25] we demonstrated that in principle the influence of the Drude extrapolation can be much decreased by considering alternative dispersion relations to Eq. (11) involving appropriate weight functions, which are aimed at suppressing the contribution of low frequencies $\omega<\omega_{\min }$, for which optical data are not available.

Let us see briefly how the more general dispersion relations come about. One starts from the following relation, generalizing Eq. (1), that can be easily proved by the contour integration method:

$$
\epsilon(i \xi)=1+\frac{2}{\pi f(i \xi)} \int_{0}^{\infty} d \omega \frac{\omega}{\omega^{2}+\xi^{2}} \operatorname{Im}[f(\omega)(\epsilon(\omega)-1)] .
$$

The above relation holds for any weight function $f(z)$, later referred to as "window" function, that is analytic in the upper complex plane, and which satisfies there the following symmetry property

$$
f\left(-z^{*}\right)=f^{*}(z),
$$

In addition, the function $f(z)$ and the permittivity $\epsilon(z)$ should be such that the quantity $u(z)=f(z)(\epsilon(z)-1)$ has at most a simple pole in the origin, and vanishes at infinity faster than $1 / z^{\alpha}$ for some $\alpha>0$. The standard Kramers-Kroning relation Eq. (1) is a special case of Eq. (9), corresponding to the choice $f(z) \equiv 1$. The distinctive feature of the general dispersion formula in Eq. (9), as contrasted to the standard KK expression Eq. (10), is that in general it involves both the real and the imaginary parts of the permittivity. This is not a problem, in principle, because both quantities can be measured, using for example ellipsometry. The key remark made in [25] was that by using window functions that vanish sufficiently fast in the origin and at infinity, it is in principle possible to strongly suppress the contribution to the integral on the r.h.s. of Eq (9) of frequencies outside the interval $\left[\omega_{\min }, \omega_{\max }\right]$ for which no optical data are available, in such a way that an accurate estimate of $\epsilon(i \xi)$ can be obtained by simply truncating the integral on the r.h.s. of Eq. (9) to the interval $\left[\omega_{\min }, \omega_{\max }\right]$. We observe that by changing the form of the window function $f(z)$ in Eq. (9), we have a means of changing at will the weight of the various spectral regions, and we can modify as well the relative weights of the real and imaginary parts of the dielectric permittivity. It is interesting to observe also that the windowed relations offer a possibility of checking the quality of the optical data, and in particular their degree of KK consistency, by verifying whether the obtained values of $\epsilon(i \xi)$ do not change when the window function is changed.

\section{A. Old form of the window functions}

As an example, in 25] we considered "window" functions $f(\omega)$ of the following form

$$
f(z)=z^{2 p+1}\left[\frac{1}{(z-w)^{2 q+1}}+\frac{1}{\left(z+w^{*}\right)^{2 q+1}}\right],
$$

where $w$ is an arbitrary complex frequency such that $\operatorname{Im}(w)<0$, and $p$ and $q$ are non-negative integers such that $0 \leq p \leq q$. A practical application of the above window functions was made in Ref. [15], where they were used to compute the permittivity $\epsilon(i \xi)$ of gold, on the basis of the tabulated handbook data [20]. The used windowed parameters were the same that had been used earlier in [25] i.e. $w=(1-2 i) \mathrm{eV} / \hbar, p=1$ and $q=3$. The obtained results were very unsatisfying, since large deviations from the KK results were found and, worse than this, unacceptable negative values for $\epsilon(i \xi)$ were found for values of $\xi$ in the interval from 2.44 to $2.92 \mathrm{eV} / \hbar$, and again for $\xi>7.8 \mathrm{eV} / \hbar$. The authors of [15] commented that the problem was presumably determined by an exceeding propagation of experimental errors affecting the optical data by the windowed relations.

After closer inspection we realized that the problem originated from the inconvenient choice of the analytic form of the window function Eq. (11) that we made in our first work. While a more systematic investigation of the problem is postponed until the next Section, we can easily understand what is wrong with the window functions in Eq. (11) by considering the quantity $g(\omega)$

$$
g(\omega)=\frac{2}{\pi f(i \xi)} \frac{\omega}{\omega^{2}+\xi^{2}} \operatorname{Im}[f(\omega)(\epsilon(\omega)-1)],
$$

whose integral along the positive real axis should reproduce, according to Eq. (9), the permittivity $\epsilon(i \xi)-1$. In Fig. 2 we plot the function $g(\omega)$, using for $\epsilon(\omega)$ the handbook data. The displayed curve corresponds to $\xi=2.5$ $\mathrm{eV} / \hbar$, and to the window parameters $p=1, q=3$ and 


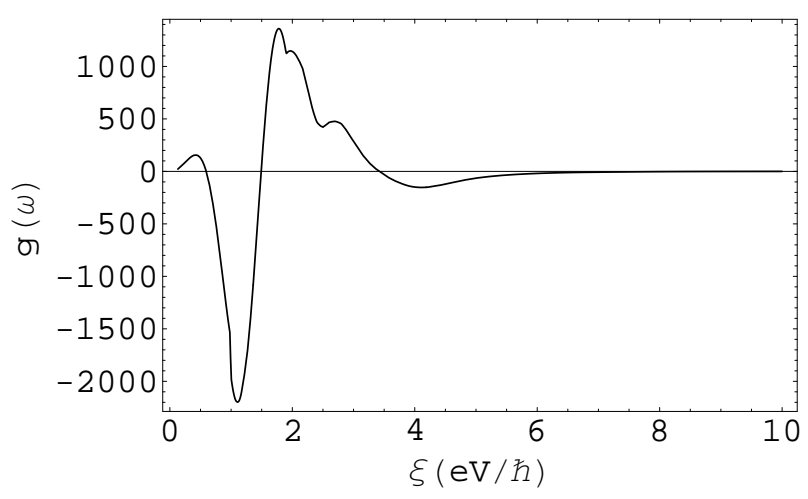

FIG. 2: Plot of the quantity $g(\omega)$ relative to the handbook optical data for gold of Ref. [20], for $\xi=2.5 \mathrm{eV} / \hbar$. The window function $f(z)$ is as in Eq. (11), for window parameters $p=1, q=3$ and $w=(1-2 i) \mathrm{eV} / \hbar$.

$w=(1-2 i) \mathrm{eV} / \hbar$. The chosen value of $\xi$ is one of those for which negative values of $\epsilon(i \xi)$ were found in [15]. We see from the Figure that $g(\omega)$ attains large positive and negative values. This implies that even slight errors in the optical data may strongly affect the delicate balance between positive and negative regions in the integral on the r.h.s. of Eq. (9), leading to large errors in the obtained value of $\epsilon(i \xi)$. That the estimates of $\epsilon(i \xi)$ are indeed very sensitive to errors in the optical data will be demonstrated more thoroughly in the next Section. Here, we remark that this instability can partially cured by using different values for the window parameter $w$, such that the quantity $g(\omega)$ displays lower peaks. However, we prefer to look for a more radical solution of the problem, by considering alternative forms of the window functions, which are presented in the next Section.

\section{B. Improved choice of the window function}

A highly desirable feature displayed by the standard KK relation Eq. (11) is that the integrand on its r.h.s. is a positive definite quantity, since the imaginary part $\epsilon^{\prime \prime}(\omega)$ of the permittivity of all materials is positive. This property of the integrand is essential in ensuring robustness of the integral with respect to small errors in the optical data, which permits to estimate reliably the permittivity $\epsilon(i \xi)$ along the imaginary frequency axis, provided only that data are available in a sufficiently large frequency interval. This led us to wonder whether there exist choices of the window function $f(z)$ such that the integrand on the r.h.s. of the generalized relations Eq. (9) has a definite sign, in such a way that the estimate of $\epsilon(i \xi)$ becomes more robust, while at the same time preserving the key feature of the generalized dispersion relations of suppressing the contribution of low frequencies, for which no optical data are available. Achieving this goal is not so easy because differently from the standard KK relation, the integrand on the r.h.s. of Eq. (9) has no definite sign in general, as it can be seen for example in Fig. 2. This is so because for window functions $f(z)$ different from one, the integrand in Eq. (9) involves both the real and the imaginary parts of the permittivity $\epsilon(\omega)$, and it is well known that differently from $\epsilon^{\prime \prime}(\omega)$, the real part of the electric permittivity $\epsilon^{\prime}(\omega)$ has no definite sign. In addition to this, the quest for an integrand of definite sign is further complicated by the fact that analyticity prevents the real and the imaginary parts of the window functions from having a definite sign, if one insists that they should vanish both at the origin and at infinity (see discussion in Ref. 25]).

Notwithstanding these general complications, fortunately enough we managed to find a class of window functions for which the integrand on the r.h.s. of Eq. (9) is in fact positive, when ohmic conductors are considered. In order for this to be possible, we had to relax the condition that the window functions vanish at infinity, and instead consider weight functions that approach one at infinity. This is not a problem at all for practical purposes, because as we said earlier optical data extend to sufficiently high frequencies for no further suppression to be necessary with the help of window functions on the high frequency side. The window functions that fit our needs have the following simple expression:

$$
f(z ; b)=\frac{z}{\sqrt{z^{2}-b^{2}}}
$$

where $b$ is an arbitrary non-negative real frequency $b \geq 0$. As we see these functions vanish in the origin, which ensures the desired suppression of low frequencies in the integral on the r.h.s. of Eq. (9). However, differently from the previous window functions in Eq. (11), these functions enjoy also the nice feature that their real and imaginary parts are both semi-definite functions along the positive frequency axis, since their imaginary part is negative for $\omega<b$ and vanishes for $\omega>b$, while their real part is zero for $\omega<b$ and positive for $\omega>b$. Interestingly, weighting functions of the form $\left(\omega^{2}-b^{2}\right)^{-1 / 2}$ have been used in the past to obtain relations connecting the refraction index $n(\omega)$ in the interval $[0, b]$ to the extinction coefficient $k(\omega)$ in the remainder of the spectrum (see Chapter 3 in [20]). Insertion of Eq. (13) into Eq. (9) results into the following formula for $\epsilon(i \xi)$ :

$$
\begin{aligned}
\epsilon(i \xi)=1+ & \frac{2}{\pi} \sqrt{1+\left(\frac{b}{\xi}\right)^{2}}\left[\int_{0}^{b} d \omega \frac{\omega^{2}}{\omega^{2}+\xi^{2}} \frac{1-\epsilon^{\prime}(\omega)}{\sqrt{b^{2}-\omega^{2}}}\right. \\
& \left.+\int_{b}^{\infty} d \omega \frac{\omega^{2}}{\omega^{2}+\xi^{2}} \frac{\epsilon^{\prime \prime}(\omega)}{\sqrt{\omega^{2}-b^{2}}}\right]
\end{aligned}
$$

We observe that the formula involves only the real part of the permittivity $\epsilon^{\prime}(\omega)$ for $\omega<b$, and only its imaginary part $\epsilon^{\prime \prime}(\omega)$ for $\omega>b$. The integrand involving $\epsilon^{\prime \prime}(\omega)$ on the r.h.s. of the above formula is positive definite, while the integrand involving $\epsilon^{\prime}(\omega)$ is positive whenever $\epsilon^{\prime}(\omega)$ is less than one. This is what we needed, because the real 


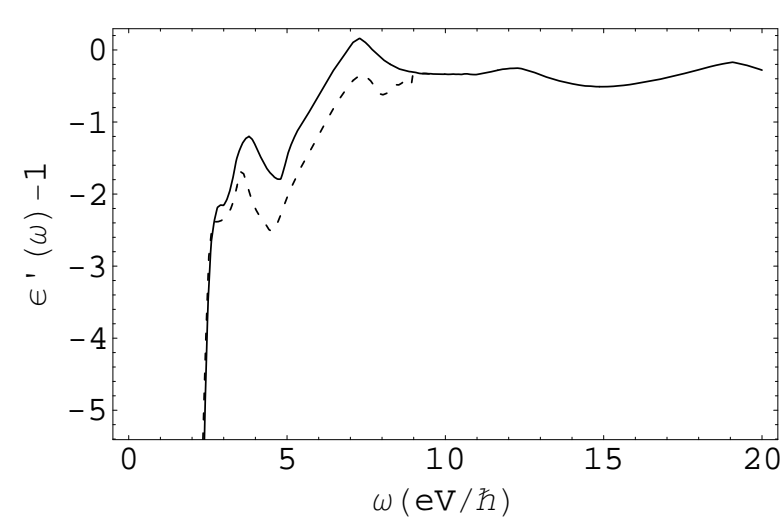

FIG. 3: Plot of $\epsilon^{\prime}(\omega)-1$ for gold. The solid line is for the handbook data of [20], the dashed one for sample 5 of Ref. [22].

part of the conductivity of ohmic conductors is characterized by the fact of being less than one essentially at all frequencies, and surely below the interband transition $\omega_{\text {inter}}$, as it it can be seen from Fig. 3. Therefore, if we choose $b$ to be any frequency such that $\epsilon^{\prime}(\omega)$ is less than one for $\omega<b$ both integrands in Eq. (14) are positive, similarly to the standard KK relations. Contrasted with the standard KK relations, the new relation Eq. (14) receives a much smaller contribution from low frequencies. This is so because in the limit of vanishing frequency the integrand in the first term of Eq. (14) vanishes like $\omega^{2}$, since $\epsilon^{\prime}(\omega)$ stays finite in the static limit, while the integrand of the standard KK relation approaches a nonvanishing constant in the same limit, due to $1 / \omega$ singularity displayed by $\epsilon^{\prime \prime}(\omega)$. Thus we may expect that the new relation permits to estimate $\epsilon(i \xi)$ more reliably than the standard KK relation because, while enjoying the robustness of a positive kernel, the weight of the Drude extrapolation is much smaller. In practical applications of Eq. (14) we shall typically consider frequencies $b$ that belong to the so-called non-relaxation region $b \gg \gamma$, and are smaller than the interband transition $\omega_{\text {inter }}$. For such values of $b$, the conditions $\epsilon^{\prime}(\omega)<0$ and $\left|\epsilon^{\prime}(\omega)\right| \gg 1$ hold, and therefore the values of $\epsilon^{\prime}(\omega)$ should not be affected by large experimental errors, contrary to what may happen at frequencies for which $\epsilon^{\prime}(\omega)$ nearly vanishes [15].

For the purpose of numerical evaluation, the occurrence of an integrable singularity for $\omega=b$ in the integrals on the r.h.s. of Eq. (14) is quite inconvenient. We can easily dispose of this singularity, however, by performing the change of variables $\omega=b \sin y$ in the first integral on the r.h.s. of Eq. (14), and $\omega=b \cosh y$ in the second one. After doing this, Eq. (14) transforms into:

$$
\epsilon(i \xi)=1+\frac{2}{\pi} \sqrt{1+\left(\frac{b}{\xi}\right)^{2}}\left[\int_{0}^{\pi / 2} d y \frac{\sin ^{2} y}{\sin ^{2} y+(\xi / b)^{2}}\right.
$$

$$
\left.\times\left[1-\epsilon^{\prime}(b \sin y)\right]+\int_{0}^{\infty} d y \frac{\cosh ^{2} y}{\cosh ^{2} y+(\xi / b)^{2}} \epsilon^{\prime \prime}(b \cosh y)\right] .
$$

\section{NUMERICAL COMPUTATIONS}

We have tested the performance of the windowed dispersion relation using the improved choice of the weight function Eq. (14) on some of the data sets for gold that have been used recently for Casimir computations. First we consider the handbook data [20]. Since they have been widely used to interpret Casimir experiments, including in particular the short-separation precise experiments [11], we shall reserve special attention to these data, and we shall spend time to evidence some inconveniences of these data that must be handled with some degree of caution. In addition to the handbook data, we used the data of the recent work 22. In principle, these data present several advantages over the handbook data. First of all, they were obtained by using ellipsometry, which is a powerful optical technique which permits to measure independently the real and the imaginary parts of the dielectric function. Since our dispersion relations explicitly involve the real part of the permittivity, it is important to have good quality data for this quantity. In addition to this, the data of [22] present two desirable features: on one hand, as we observed earlier, the data in 22] extend down to a frequency $\omega_{\min }=38 \mathrm{meV} / \hbar$, which is lower than the minimum frequency $\omega_{\min }=0.125 \mathrm{eV} / \hbar$ reached by the handbook data. We shall find indeed that a lower frequency around $40 \mathrm{meV} / \hbar$ is sufficient to compute with high precision the Casimir force, using the windowed dispersion relations. The second and perhaps more important feature of Ref. [22] is that it provides homogeneous data on well defined gold samples over the wide range of wavelengths from 0.14 to $33 \mu \mathrm{m}$, which give the main contribution to the Casimir effect. This is not so for the handbook [20], whose data in the important spectral region from the near IR to the near UV combine results from two different experiments [29, 30], utilizing differently prepared gold films. We shall see later on that the optical properties of these gold films display striking differences, and therefore wrong results may easily result if the handbook data are used in computations where KK consistency of the data plays an important role.

\section{A. Suppression of the Drude contribution}

Our key motivation for introducing windowed dispersion relations is that they strongly reduce the weight of the Drude extrapolation, and correspondingly increase the weight of experimental optical data, in such a way that obtained values for $\epsilon(i \xi)$ are much less sensitive to experimental uncertainties in the Drude parameters. We can verify to what extent the new windowed relation Eq. (14) suppresses the contribution of the Drude extrapo- 


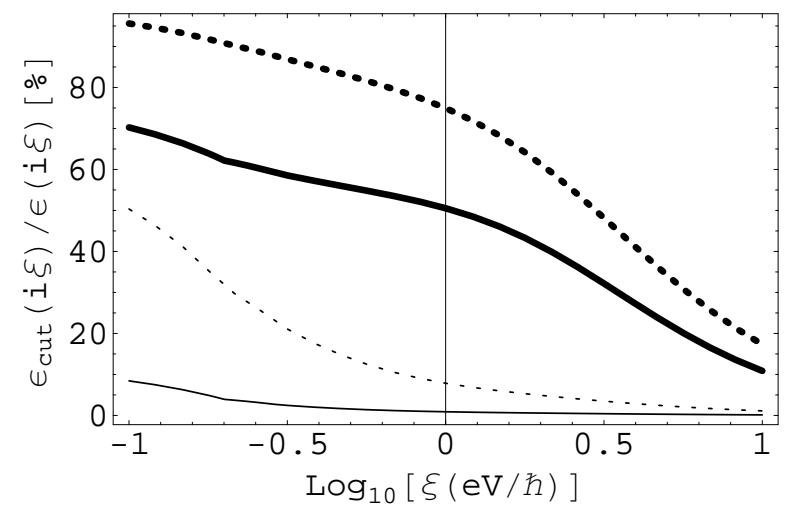

FIG. 4: Relative contribution of the Drude extrapolation $\epsilon_{\text {cut }}(i \xi)$ to the dielectric function $\epsilon(i \xi)$, according to the standard KK formula (thick lines) and to the windowed formula Eq. (14) (thin lines). Solid lines were computed using optical data of sample 5 of Ref. [22] (solid lines), while the dashed lines were computed using the handbook data [20].

lation by proceeding in a way similar to Ref. [22]. We decompose the quantity $\epsilon(i \xi)$ in Eq. (9) in a way analogous to Eq. (3):

$$
\epsilon(i \xi)=1+\epsilon_{\mathrm{cut}}(i \xi ; b)+\epsilon_{\operatorname{expt}}(i \xi ; b)
$$

where $\epsilon_{\text {cut }}(i \xi ; b)$ is calculated using the Drude extrapolation for $\epsilon(\omega)$ in the unaccessible frequency range $\omega<$ $\omega_{\text {min }}$, while $\epsilon_{\text {expt }}(i \xi ; b)$ is calculated using the experimental optical data, according to the following formulas:

$$
\begin{gathered}
\epsilon_{\text {cut }}(i \xi ; b)= \\
\frac{2}{\pi f(i \xi ; b)} \int_{0}^{\omega_{\min }} d \omega \frac{\omega}{\omega^{2}+\xi^{2}} \operatorname{Im}\left[f(\omega ; b)\left(\epsilon_{\operatorname{Dr}}(\omega)-1\right)\right]
\end{gathered}
$$

and

$$
\begin{gathered}
\epsilon_{\mathrm{expt}}(i \xi ; b)= \\
\frac{2}{\pi f(i \xi)} \int_{\omega_{\min }}^{\infty} d \omega \frac{\omega}{\omega^{2}+\xi^{2}} \operatorname{Im}\left[f(\omega ; b)\left(\epsilon_{\operatorname{expt}}(\omega)-1\right)\right]
\end{gathered}
$$

where $f(z ; b)$ is as in Eq. (13). In order to demonstrate how well the windowed formula suppresses the Drude contribution $\epsilon_{\text {cut }}(i \xi)$, in Fig. \$ we plot the relative contribution of the Drude extrapolation $\epsilon_{\text {cut }}(i \xi) / \epsilon(i \xi)$, that obtains if one uses the standard KK relation (thick upper solid and dashed lines) or the generalized dispersion relation (thin lower solid and dashed lines) with $f(z ; b)$ given by Eq. (13), for $b=1 \mathrm{eV} / \hbar$. The displayed imaginaryfrequency range extending from $0.1 \mathrm{eV} / \hbar$ to ten $\mathrm{eV} / \hbar$ is the one relevant for determining the Casimir force for plates separations larger than $100 \mathrm{~nm}$. The solid lines in Fig. 4 were computed using the optical data relative to sample 5 of Ref. 22], while the dashed lines are relative to the handbook data 20]. We recall that in [22] optical data were measured in the frequency interval extending from $\omega_{\min }=0.042 \mathrm{eV} / \hbar$ to $\omega_{\max }=9 \mathrm{eV} / \hbar$, and so for $\omega$ larger than $9 \mathrm{eV} / \hbar$ we used data from Ref. [20] to compute $\epsilon_{\text {expt }}(i \xi)$. The Drude parameters used to compute $\epsilon_{\text {cut }}(i \xi)$ were as follows: for sample 5 of [22] we used the average values quoted there, i.e. $\omega_{p}=8.38 \mathrm{eV} / \hbar$ and $\gamma=37.1 \mathrm{meV} / \hbar$, while for the handbook data we used the commonly used values $\omega_{p}=9 \mathrm{eV} / \hbar$ and $\gamma=35$ $\mathrm{meV} / \hbar$. It is apparent from Fig. 4 that the generalized dispersion relation is very effective in suppressing the contribution of the Drude extrapolation, in comparison to the standard KK relation. This is especially so for the data of 22], due to the fact that they extend to smaller frequencies than the handbook data. In this case, for the lowest displayed imaginary frequency of $0.1 \mathrm{eV} / \hbar$, the relative contribution of $\epsilon_{\text {cut }}$ is reduced from $70.2 \%$ to $8.4 \%$, but already for $\xi=0.5 \mathrm{eV} / \hbar$ it is reduced from 55 $\%$ to $1.5 \%$, and it becomes rapidly negligible for larger imaginary frequencies.

We can show that thanks to suppression of the Drude extrapolation, experimental uncertainties in the Drude parameters lead to much smaller uncertainties in the dielectric function $\epsilon(i \xi)$, when the window method is used in comparison with the standard KK formula. This in turn implies that the Casimir force can be predicted more accurately by the widow approach. This is an important result because experimental uncertainties in the Drude parameters have a non-negligible impact on the Casimir force, when standard KK relations are used. To be definite, we considered the optical data of [22]. In this work the Drude parameters for the used gold films were determined by several methods, and precisely by fitting with the Drude formula both $\epsilon(\omega)$ and the complex refraction index $n(\omega)=\sqrt{\epsilon(\omega)}$, and also by performing a KK analysis of the data. Depending on the used method, the obtained values of $\omega_{p}$ and $\gamma$ for the five available gold samples showed non-negligible variations, ranging from a minimum of $1 \%$ to a maximum of $2 \%$ for $\omega_{p}$, and from $5 \%$ to $14 \%$ for $\gamma$. It was found that by itself this uncertainty in the Drude parameters entails an uncertainty in the Casimir force that can reach $1 \%$ at separations around $100 \mathrm{~nm}$. In order to demonstrate the effectiveness of the window method in reducing this source of uncertainty, in Fig. 5 we plot the relative per cent variation of the dielectric function $\epsilon(i \xi)$ determined by small changes in the Drude parameters, according to the standard KK formula (thick lines) and to the windowed formula Eq. (14) (thin lines). The upper four lines correspond to a $2 \%$ increase in $\omega_{p}$, while the four lower lines correspond to a $14 \%$ increase of $\gamma$. Solid lines were computed using optical data for sample 4 of Ref. [22] (solid lines), while the dashed lines were computed using the handbook data [20]. In Fig. 6 we display the corresponding per cent variations in the Casimir pressure between two gold plates in vacuum, at room temperature. The lines in Fig. 6 have the same meanings an in Fig. 5, apart from the fact that now the 4 lower curves correspond to a $2 \%$ increase in $\omega_{p}$, while the four upper curves correspond to a $14 \%$ 


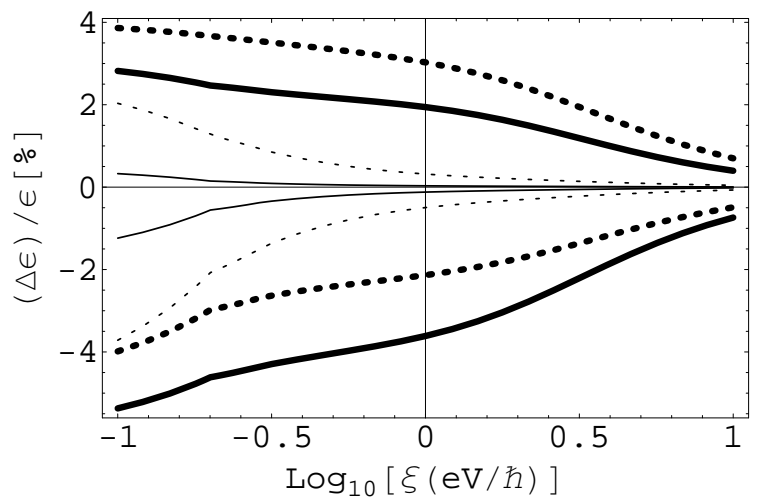

FIG. 5: Relative per cent variation of the dielectric function $\epsilon(i \xi)$ determined by small changes in the Drude parameters, according to the standard KK formula (thick lines) and to the windowed formula Eq. (14) (thin lines). The upper four lines correspond to a $2 \%$ increase in $\omega_{p}$, while the four lower lines correspond to a $14 \%$ increase of $\gamma$. Solid lines were computed using optical data of sample 4 of Ref. [22] (solid lines), while the dashed lines were computed using the handbook data [20].

increase of $\gamma$. We clearly see that uncertainties in the Drude parameters have a much smaller impact when the window approach is used, as compared to the traditional $\mathrm{KK}$ relations. It is also clear that the window method is more effective when applied to the data of 22] than for the handbook data. Indeed we see that when the former data are used, the maximum uncertainty in the Casimir pressures decreases from about $1 \%$ to $0.05 \%$, while for the handbook data the maximum uncertainty decreases

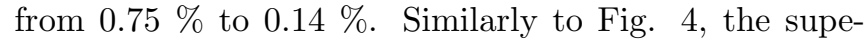
rior performance of the window method when the data of 22] are used is a consequence of the fact that these data extend to smaller frequencies than the handbook data.

\section{B. Propagation of random errors}

The next thing that we tested is how possible errors in the optical data are propagated by windowed dispersion relations. Indeed, if we could rely on perfect optical data with no uncertainties, the mathematical properties of analytic functions would permit to compute the exact values of the permittivity anywhere else in the upper complex plane, starting from knowledge of the dielectric permittivity in any however small interval of the real frequency axis. The existence of experimental errors in the optical data makes this goal impossible to achieve, and in practice if one tries to compute the dielectric function at points of the complex plane that are too far for the experimental data, one inevitably ends up with estimates having large errors. In view of this practical limitation, it is important to investigate quantitatively how uncertainties in the optical data are propagated by the dispersion formulae used to compute $\epsilon(i \xi)$. This is not an easy ques-

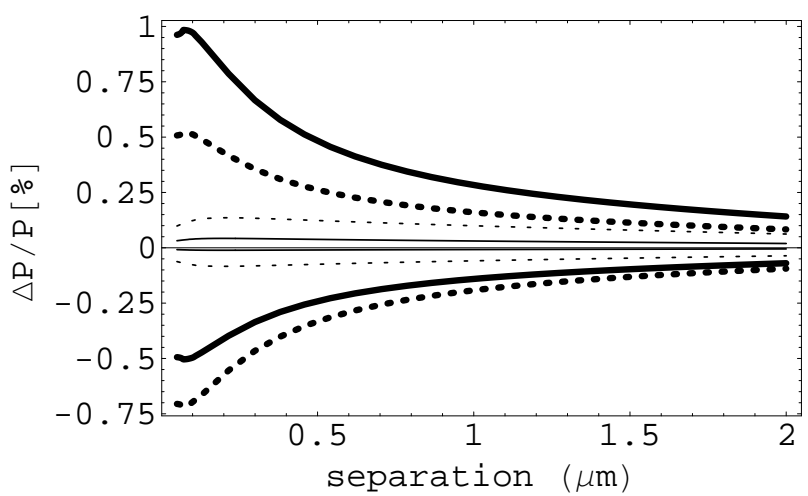

FIG. 6: Relative per cent variation of the Casimir pressure between two gold plates at room temperature, determined by small changes in the Drude parameters, according to the standard KK formula (thick lines) and to the windowed formula Eq. (14) (thin lines). The lower four lines correspond to a 2 $\%$ increase in $\omega_{p}$, while the four upper lines correspond to a $14 \%$ increase of $\gamma$. Solid lines were computed using optical data of sample 4 of Ref. [22] (solid lines), while the dashed lines were computed using the handbook data [20].

tion to address, because a complete answer can be given only if sufficient information is provided on the nature the experimental errors affecting the data.

Since neither the handbook [20] nor Ref. [22] provide detailed information on the magnitude of the experimental errors affecting their data, we performed a Monte Carlo simulation to estimate how statistical errors in the optical data are propagated by the windowed dispersion relations, under the reasonable assumption of a statistical uncertainty of a few per cent in the optical data. We proceeded as follows. Optical data are usually presented as lists of values $\mathcal{S} \equiv\left\{n_{\exp }\left(\omega_{i}\right), k_{\exp }\left(\omega_{i}\right)\right\}, i=1, \ldots N$ for the refraction index $n(\omega)$ and the extinction coefficient $k(\omega)(n(\omega)+i k(\omega)=\sqrt{\epsilon(\omega)})$, for a discrete set of frequencies $\omega_{i}: \omega_{\min } \equiv \omega_{1}<\omega_{2}<\ldots<\omega_{N} \equiv \omega_{\max }$. For any definite set $\mathcal{S}$ of data, say the handbook data, we computed the quantity $\epsilon_{\text {expt }}(i \xi)$ using the generalized formula in Eq. (18). We then made the simple assumption that the experimental values of $n$ and $k$ in $\mathcal{S}$ had a common statistical per cent error $\delta_{\text {exp }}$. In order to estimate the corresponding error $\delta \epsilon_{\text {expt }}(i \xi)$, we randomly generated $M$ new hypothetical sets of data $\mathcal{S}_{\alpha}=$ $\left\{\left(n_{1}^{(\alpha)}, k_{1}^{(\alpha)}\right), \cdots,\left(n_{N}^{(\alpha)}, k_{N}^{(\alpha)}\right)\right\}, \alpha=1,2, \cdots M$. Each data set $\mathcal{S}_{\alpha}$ was generated by extracting $2 N$ random numbers $\left\{n_{i}, k_{i}\right\}, i=1 \ldots N$ from $2 N$ independent gaussian distributions, having mean values respectively equal to the experimental values $\left\{n_{\exp }\left(\omega_{i}\right), k_{\exp }\left(\omega_{i}\right)\right\}$, and variances respectively equal to $\left\{\left(n_{\exp }\left(\omega_{i}\right) \delta_{\exp }\right)^{2},\left(k_{\exp }\left(\omega_{i}\right) \delta_{\exp }\right)^{2}\right\}$. Each data set $\mathcal{S}_{\alpha}$ was then used to obtain a new estimate $\epsilon_{\text {expt }}^{(\alpha)}(i \xi)$ and the average (absolute) error $\delta \epsilon_{\text {expt }}(i \xi)$ on 


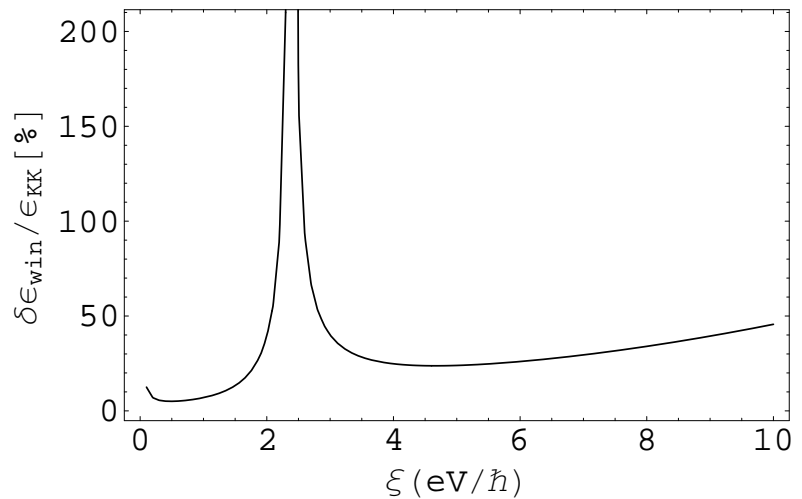

FIG. 7: Simulated uncertainty (in per cent) in the value of the dielectric function $\epsilon(i \xi)$ versus imaginary frequency (in $\mathrm{eV} / \hbar)$. The dielectric permittivity $\epsilon(i \xi)$ was computed using the handbook data [20], with the help of the windowed dispersion relation for the choise of window functions in Eq. (11). The used window parameters are $p=1, q=3$ and $w=(1-2 i) \mathrm{eV} / \hbar$. In the simulation we assumed a $3 \%$ error in both $n(\omega)$ and $k(\omega)$.

$\epsilon_{\text {expt }}(i \xi)$ was estimated by the formula:

$$
\delta \epsilon_{\mathrm{expt}}(i \xi)=\sqrt{\frac{1}{M-1} \sum_{\alpha=1}^{M}\left[\epsilon_{\mathrm{expt}}^{(\alpha)}(i \xi)-\epsilon_{\mathrm{expt}}(i \xi)\right]^{2}} .
$$

In our simulation we took $M=1000$. We studied by this method both the old window functions Eq. (11), and the new ones Eq. (13).

We recall that by using the windowed relations with window functions of the form given in Eq. (11) the authors of [15] obtained unacceptable negative values for $\epsilon(i \xi)$ in certain imaginary frequency ranges, and also for the frequencies for which positive results were found, large deviations from the KK values were observed. In order to explain the findings of [15], we investigated first these window functions, for the same values of the window parameters $p=1, q=3$ and $w=(1-2 i) \mathrm{eV} / \hbar$ that were used in our earlier work [25], and that were subsequently considered by Geyer et. al [15]. Results of the Monte Carlo simulations give support to the conjecture made by the authors of [15], that this choice of the window function determines an exceeding amplification of experimental errors in the optical data, explaining the obtained negative values for $\epsilon(i \xi)$. This can be seen from Fig. 7, where we plot the fractional random error $\delta \epsilon_{\text {expt }}(i \xi) / \epsilon_{\mathrm{KK}}(i \xi)$ (in per cent) for the above choice of window parameters, assuming a $3 \%$ error in both $n(\omega)$ and $k(\omega)$. As we see, the error $\delta \epsilon_{\text {expt }}(i \xi)$ is very large at all frequencies. The largest error occurs for values of $\xi$ near the zero $\xi_{0}=2.4$ displayed by the window function along the imaginary axis. It is clear that estimates of $\epsilon(i \xi)$ obtained with this choice of the window function do not have much meaning. We verified that better results can be obtained for different values of the window parameter $w$. For example, for $w=-5 i \mathrm{eV} / \hbar$ the un-

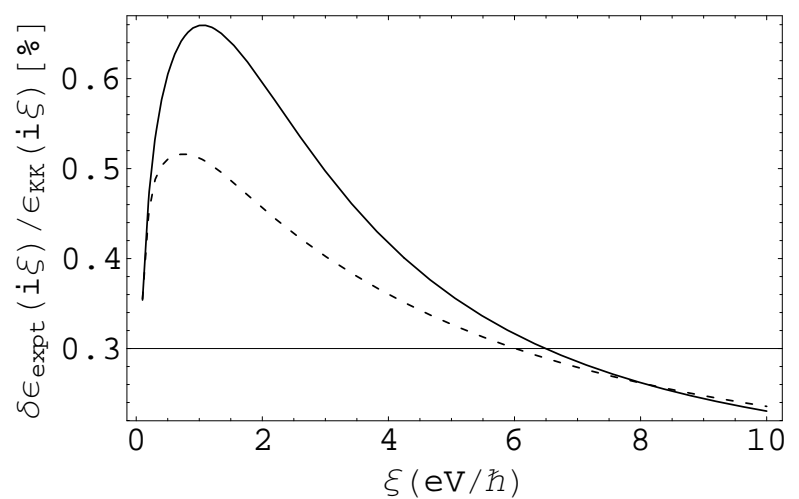

FIG. 8: Simulated uncertainty (in per cent) in the value of the dielectric function $\epsilon(i \xi)$ versus imaginary frequency (in $\mathrm{eV} / \hbar)$. The dielectric permittivity $\epsilon(i \xi)$ was computed using the handbook data [20], with the help of the windowed dispersion relation Eq. (14), for $b=1 \mathrm{eV} / \hbar$ (solid line) and for $b=3 \mathrm{eV} / \hbar$ (dashed line). In the simulation we assumed a 3 $\%$ error in both $n(\omega)$ and $k(\omega)$.

certainty $\delta \epsilon_{\text {expt }}(i \xi)$ becomes less than 7 per cent, in the entire range of $\xi$ from 0.1 and $10 \mathrm{eV} / \hbar$, again assuming a $3 \%$ error in both $n(\omega)$ and $k(\omega)$.

Even though it is possible to sensibly reduce the errors by suitably tuning the value of $w$, in a $\xi$-dependent way, we did not pursue further the old form of the window functions, because much better results can be obtained by using the new window functions given in Eq. (13), and the associated dispersion relation Eq. (14). With such a choice of the window function, the uncertainty in $\epsilon_{\text {expt }}(i \xi)$ decrease tremendously. This is shown in Fig. 8 , where we plot the fractional uncertainty $\delta \epsilon_{\text {expt }}(i \xi) / \epsilon_{\mathrm{KK}}(i \xi)$ (in per cent), versus the imaginary frequency $\xi$ (in $\mathrm{eV} / \hbar$ ), for the two values of the window parameter $b=1 \mathrm{eV} / \hbar$ (solid line) and $b=3 \mathrm{eV} / \hbar$ (dashed line), again assuming a 3 $\%$ error in the handbook data for $n(\omega)$ and $k(\omega)$. We see that the uncertainty $\delta \epsilon_{\text {expt }}(i \xi) / \epsilon_{\mathrm{KK}}(i \xi)$ is now less than $0.7 \%$ for all displayed imaginary frequencies. Thus our Monte Carlo simulation gives support to the expectation that the new window functions permit to obtain robust estimates of the dielectric function $\epsilon(i \xi)$, thanks to the positive definite character of the corresponding integrand on the r.h.s. of Eq. (9). In view of these findings, we shall not consider any longer the old window functions in the next Sections, and we shall exclusively rely on the new ones for our next computations.

\section{AN APPLICATION TO TABULATED DATA FOR GOLD}

In this Section we shall use the new window functions and the associated dispersion relation Eq. (14) to obtain an estimate of dielectric permittivity $\epsilon(i \xi)$ of gold, on the basis of the handbook data [20].

Before we do it, an important observation on the con- 
TABLE I: Values of the complex permittivity for gold films from Ref. [29] and Ref. [30], that have been both included in the handbook [20].

\begin{tabular}{|c|c|c|}
\hline \hline$\omega($ in eV $/ \hbar)$ & $\epsilon(\omega)$ (Ref. [29]) & $\epsilon(\omega)($ Ref. [30]) \\
\hline 0.6 & $-168.2+\mathrm{i} 23.3$ & $-170.7+\mathrm{i} 25.6$ \\
0.7 & $-125.0+\mathrm{i} 15.6$ & $-165.9+\mathrm{i} 18.7$ \\
0.8 & $-96.0+\mathrm{i} 11.0$ & $-131.9+\mathrm{i} 12.65$ \\
0.9 & $-76.7+\mathrm{i} 7.96$ & $-94.3+\mathrm{i} 8.9$ \\
\hline \hline
\end{tabular}

sistency of these data is in order. The handbook 20] quotes values for the refraction index $n(\omega)$ and the extinction coefficient $k(\omega)$ of gold in the frequency range from $\omega_{\min }=0.125 \mathrm{eV} / \hbar$ up to $\omega_{\max }=9919 \mathrm{eV} / \hbar$. An important feature of these data is that they collect together results from different experiments, performed in different spectral regions, utilizing gold films prepared by different procedures. In particular, data in the infrared region of the spectrum, with frequencies in the interval $0.125 \mathrm{eV} / \hbar \leq \omega \leq 0.98 \mathrm{eV} / \hbar$, were taken from Ref.[29], which used an evaporated gold film on a polished glass substrate. Data in the frequency region $0.6 \mathrm{eV} / \hbar \leq \omega \leq 6 \mathrm{eV} / \hbar$ were taken from Ref. [30], which used annealed films evaporated in ultrahigh vacuum on fused silica substrates. Data in the interval $6.199 \mathrm{eV} / \hbar \leq \omega \leq 26.38 \mathrm{eV} / \hbar$ were taken from Ref. [31], which used evaporated films onto polished glass substrates, in a conventional vacuum system. Finally, data in the interval $26 \mathrm{eV} / \hbar \leq \omega \leq 88 \mathrm{eV} / \hbar$ were taken from Ref. [32], which used thin gold films evaporated onto substrates of collodion. For the purpose of Casimir computations, the important spectral regions are the IR one covered by Dold's et al. data [29], and the region extending from the near IR to the near UV in Theye's data [30]. At a closer inspection, one realizes that these two sets of data are quite inconsistent with each other, probably because of the different deposition procedures adopted in these measurements. This can be appreciated by comparing the values, presented in Table 1, of the dielectric function $\epsilon(\omega)$ computed from the two sets of data, in the spectral region where they overlap i.e. from $0.6 \mathrm{eV} / \hbar$ to $0.9 \mathrm{eV} / \hbar$. We clearly see that there exist large differences between the two samples.

A more striking measure of the inconsistency between the two data sets is obtained by estimating the respective plasma frequencies $\omega_{p}$. This is an important quantity to consider, because as we saw earlier its value has a large impact on the magnitude of the Casimir force. We have estimated $\omega_{p}$ by the following procedure. It is well known [33] that for frequencies $\omega$ in the non-relaxation region $\omega \gg \gamma$ but smaller than the interband transition $\omega_{\text {inter }}\left(\omega_{\text {inter }} \simeq 2 \mathrm{eV} / \hbar\right.$ for gold $)$, the real part of the electric permittivity of metals can be well described by the formula:

$$
\epsilon^{\prime}=1+\epsilon_{\text {inter }}-\frac{\omega_{p}^{2}}{4 \pi^{2} c^{2}} \lambda^{2},
$$

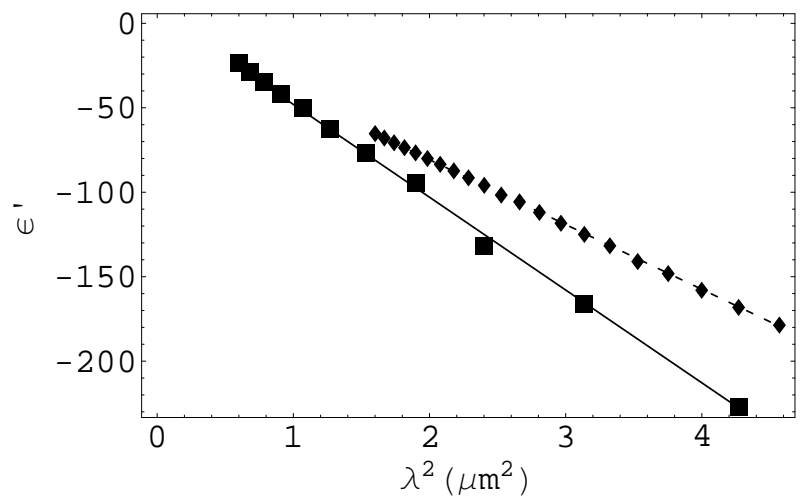

FIG. 9: Real part of the dielectric function of gold in the infrared and red regions, versus square of wavelength (with $\lambda$ in microns). Displayed data are from Ref. [30] (squares) and [29] (diamond), which are part of the handbook 20]. Also displayed are the linear fits to the data (solid and dashed lines), according to Eq. (20). The used fit parameters for the plasma frequency are $\omega_{p}=9.19 \mathrm{eV} / \hbar$ (solid line) and $\omega_{p}=7.7 \mathrm{eV} / \hbar$ (dashed line line).

where $\lambda$ is the wavelength and $\epsilon_{\text {inter }}$ is the contribution of interband transitions, which for frequencies sufficiently below $\omega_{\text {inter }}$ can be considered as a constant. From the above relation, we expect that $\epsilon^{\prime}$ should vary linearly with the $\lambda^{2}$, with a slope proportional to $\omega_{p}^{2}$. We determined $\omega_{p}$ by fitting the data in the infrared and red regions of the spectrum. Since Theye's data [30] extend to the interband region, we did not use $\epsilon_{\text {inter }}$ as a fit parameter for these data, but we computed its value by means of the dispersion formula:

$$
\epsilon_{\text {inter }}=\frac{2}{\pi} \int_{\omega_{\text {inter }}}^{\infty} \frac{d \omega}{\omega} \epsilon^{\prime \prime}(\omega)
$$

from which we obtained $\epsilon_{\text {inter }}=5.9$. In the case Dold's et al. data [29], $\epsilon_{\text {inter }}$ was used as a fit parameter. We note however that the values of $\epsilon_{\text {inter }}$ have little impact on the obtained values of the plasma frequency $\omega_{b}$ because for the considered wavelengths $\left|\epsilon^{\prime}(\omega)\right| \gg \epsilon_{\text {inter. }}$. The fits resulted in the following values of the plasma frequency for the two sets of data: $\omega_{p}=9.19 \mathrm{eV} / \hbar$ for Theye's data and $\omega_{p}=7.7 \mathrm{eV} / \hbar$ for Dold's et al. data. The data and the fitting straight lines are displayed in Fig. 9, from which we see that the linear fits are very good for both sets of data. The obtained value of $\omega_{p}$ for Theye's data compares well with the value of $9 \mathrm{eV} / \hbar$ that was obtained on theoretical grounds by Lambrecht and Reynaud [24], as well as with the value of $8.9 \mathrm{eV} / \hbar$ that was obtained by the authors of 11] from resistivity measurements on their gold samples. The value of $\omega_{p}$ that we obtained for Dold's et al data is consistent with the value of $7.5 \mathrm{eV} / \hbar$ that was obtained by a similar method in Ref. [23]. In the latter work however no attempt was made to determine the plasma frequency for Theye's data separately.

Since the value of the plasma frequency for Dold's data is so much smaller than that for Theye's data, we have de- 


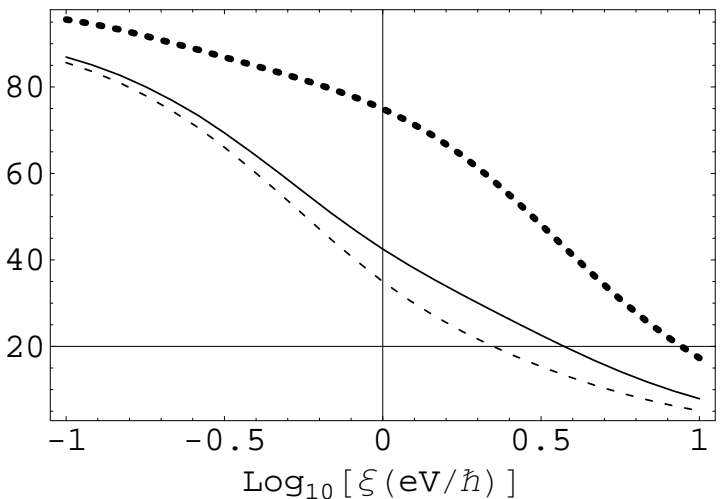

FIG. 10: Relative contribution of the Drude extrapolation $\epsilon_{\text {cut }}(i \xi)$ to the dielectric function $\epsilon(i \xi)$ of gold, for the standard KK relation (thick dotted line) using the complete handbook data $\left(\omega_{\min }=0.125 \mathrm{eV} / \hbar\right)$, and the windowed dispersion formula after exclusion of Dold's et al. data [29] $\left(\omega_{\min }=0.6\right.$ $\mathrm{eV} / \hbar$ ) for $b=1 \mathrm{eV} / \hbar$ (thin solid line) and $b=1.5 \mathrm{eV} / \hbar$ (thin dashed line).

cided not to use Dold's data in our computation of the dielectric function along the imaginary frequency axis. The minimum frequency $\omega_{\min }$ of the remaining data therefore coincides with the one $\omega_{\min }=0.6 \mathrm{eV} / \hbar$ for Theye's data. The Drude parameters used to extrapolate the data below $0.6 \mathrm{eV} / \hbar$ were same as in [11], i.e. $\omega_{p}=8.9 \mathrm{eV} / \hbar$ and $\gamma_{p}=35.7 \mathrm{meV} / \hbar$. The price we had to pay for excluding Dols'd data is that the contribution of the Drude extrapolation returns to be significant, even if the windowed dispersion relation Eq. (14) is used, as it can be seen by comparing the thin lines in Fig. 10 with the dotted thick line in Fig. 4. However, thanks to the ability of the windowed relation to suppress low frequencies, we see that despite the large value of $\omega=0.6 \mathrm{eV} / \hbar$, the weight of the Drude extrapolation in the windowed relation is significantly less than the one (thick dotted line in Fig. 10) resulting from the full set of tabulated data, beginning from $0.125 \mathrm{eV} / \hbar$, when the traditional KK relation is used.

We compared the values of $\epsilon(i \xi)$ obtained from Eq. (14) with those obtained from the standard KK relation Eq. (11). We shall denote the two estimates by $\epsilon_{\mathrm{win}}(i \xi)$ and $\epsilon_{\mathrm{KK}}(i \xi)$, respectively. In Fig. 11 we display the fractional difference $\left(\epsilon_{\text {win }}(i \xi)-\epsilon_{\mathrm{KK}}(i \xi)\right) / \epsilon_{\mathrm{KK}}(i \xi)$ (in percent) between the two estimates. The solid and the dashed lines are for $b=1 \mathrm{eV} / \hbar$, and for $b=1.5 \mathrm{eV} / \hbar$, respectively. We remark that the standard $\mathrm{KK}$ values of $\epsilon_{\mathrm{KK}}(i \xi)$ in Fig. 11 have been computed using the full handbook's data, and therefore they coincide with the values used in [11] for comparison of the Drude model approach with experiments. We note that the windowed values of $\epsilon(i \xi)$ obtained for the two chosen values of the window parameter $b$ differ from each other by less than one per cent, and both differ from the KK values by roughly the same amount. So we see that the improved window method produces results that are substantially

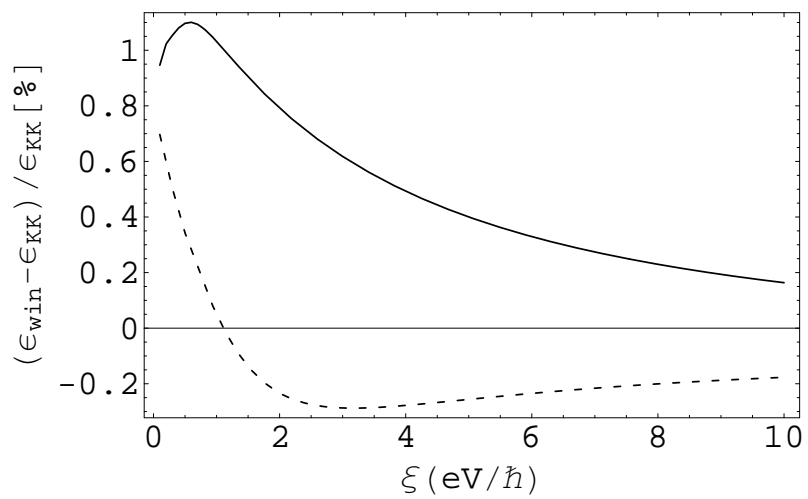

FIG. 11: Per cent difference between the window and the KK estimates for the permittivity $\epsilon(i \xi)$ of gold, computed using the handbook data [20]. The windowed estimate was computed using Eq. (13), after excluding Dold's data, for $b=1 \mathrm{eV} / \hbar$ (solid line) and $b=1.5 \mathrm{eV} / \hbar$ (dashed line). The Drude parameters used to extrapolate the data are $\omega_{p}=8.9$ $\mathrm{eV} / \hbar$ and $\gamma=35.7 \mathrm{meV} / \hbar$.

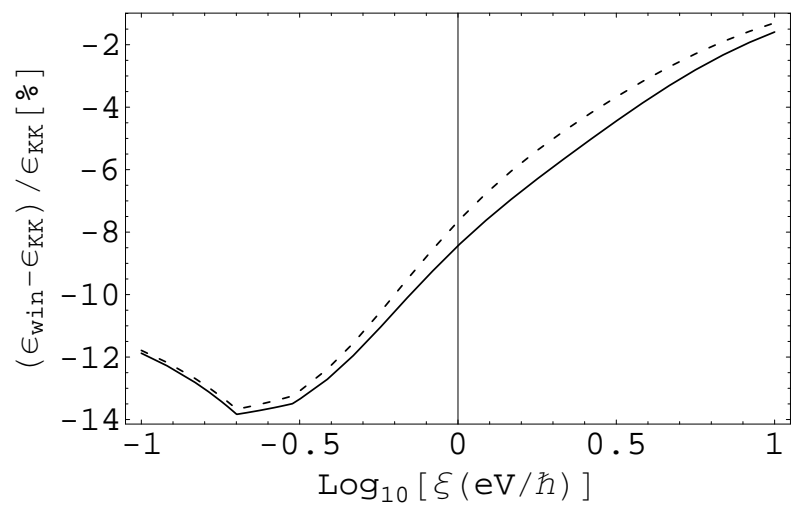

FIG. 12: Per cent difference between the window and the KK estimates for the permittivity $\epsilon(i \xi)$ of gold, both computed using the full handbook data [20]. The windowed estimate was computed using Eq. (13), with $b=1 \mathrm{eV} / \hbar$ (solid line) and $b=1.5 \mathrm{eV} / \hbar$ (dashed line). The Drude parameters used to extrapolate the data are $\omega_{p}=8.9 \mathrm{eV} / \hbar$ and $\gamma=35.7$ $\mathrm{meV} / \hbar$.

consistent with the traditional method. We think however that the window estimates obtained here are nevertheless more reliable than the traditional ones, because they rely to a less extent on the Drude extrapolation.

It is interesting to see how the above results change if we use the window method with the full set of handbook's data. This can be seen from Fig. 12 where we plot the corresponding fractional difference $\left(\epsilon_{\text {win }}(i \xi)-\right.$ $\left.\epsilon_{\mathrm{KK}}(i \xi)\right) / \epsilon_{\mathrm{KK}}(i \xi)$ (in per cent), the solid and the dashed lines have the same meaning as in Fig. 11. It is evident that after we include Dold's data the agreement between the window and the KK estimates worsens significantly. We interpret this fact as a further proof of the KK inconsistency that results from combination of Theye's and Dold's data. 


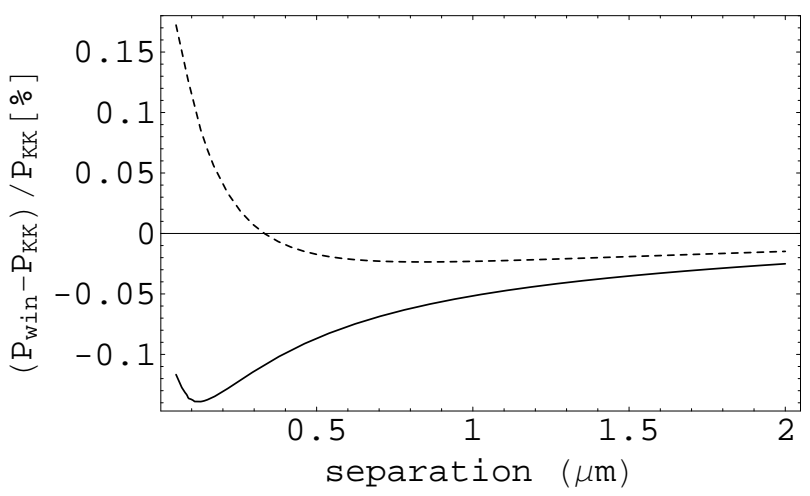

FIG. 13: Per cent difference between the window and the KK estimates for the Casimir pressure between two gold plates, computed using the handbook data [20]. The windowed pressures $P_{\text {win }}$ were computed excluding Dold's data, by using Eq. (13) for $b=1 \mathrm{eV} / \hbar$ (solid line) and $b=1.5 \mathrm{eV} / \hbar$ (dashed line). The Drude parameters used to extrapolate the data are $\omega_{p}=8.9 \mathrm{eV} / \hbar$ and $\gamma=35.7 \mathrm{meV} / \hbar$.

We used the windowed estimates of $\epsilon(i \xi)$ to compute the Casimir pressure between two parallel gold plates in vacuum. We let $P_{\text {win }}(a, T)$ and $P_{\mathrm{KK}}(a, T)$, respectively, the Casimir pressure obtained by plugging into Eq. (6) the windowed and the traditional KK estimates for the permittivity $\epsilon(i \xi)$. In Fig. 13 we plot the per cent difference $\left[P_{\text {win }}(a, T)-P_{\mathrm{KK}}(a, T)\right] / P_{\mathrm{KK}}(a, T)$ between the windowed and the KK pressures computed using the handbook data, versus plate separation (in microns). As before, the windowed values were computed excluding Dold's data, while the KK pressures use the full set of data. Two different values of the window parameter $b$ were considered, namely $b=1 \mathrm{eV} / \hbar$ (solid line) and $b=1.5 \mathrm{eV} / \hbar$ (dashed line). As we see, the pressures computed by the window method differ from those obtained by the traditional KK method by less than $0.2 \%$ in the entire displayed separation range. The width of the region comprised between the solid and the dashed lines in Fig. 11 provides an indication of the uncertainty in the windowed prediction of the Casimir pressure, if the handbook data are used. We estimate it to be less than $0.3 \%$ for separations larger than $50 \mathrm{~nm}$.

\section{APPLICATION TO OPTICAL DATA OF REF. 22]}

We apply now the windowed dispersion relation Eq. (14) to some of the optical data for gold films of Ref. [22]. As we discussed at the beginning of Sec. IV these data are particularly interesting for our purposes, because the window method is expected to provide very reliable results for the dielectric permittivity $\epsilon(i \xi)$ of these samples, thanks to the fact that in [22] both the real and the imaginary parts of $\epsilon(\omega)$ were measured independently by ellipsometry over the wide range of frequencies from
TABLE II: Values of the Drude parameters for samples 1, 4 and 5 of Ref. 22].

\begin{tabular}{|c|c|c|}
\hline \hline Sample & $\omega_{p}[\mathrm{eV} / \hbar]$ & $\gamma[\mathrm{meV} / \hbar]$ \\
\hline 1 & $6.82 \pm 0.08$ & $40.5 \pm 2.1$ \\
4 & $8.00 \pm 0.16$ & $35.7 \pm 5.1$ \\
5 & $8.38 \pm 0.08$ & $37.1 \pm 1.9$ \\
\hline \hline
\end{tabular}

$35 \mathrm{meV} / \hbar$ to about $9 \mathrm{eV} / \hbar$, which includes longer wavelengths than the tabulated data. Of the five gold samples described in [22], we considered samples 1, 4 and 5 . Samples 1 and 4 consisted of gold films deposited on a Si substrate, with thicknesses of $400 \mathrm{~nm}$ and $120 \mathrm{~nm}$ respectively, while sample 5 consisted of an annealed gold film with a thickness of $120 \mathrm{~nm}$ deposited on a cleaved mica substrate. The Drude parameters for these films were determined in [22] by several different methods. For the convenience of the reader they are reported in Table II. We note that samples 1 and 5 (see Table II of [22]) are characterized, respectively, by having the smallest and largest values of the plasma frequency $\omega_{p}$, and therefore the corresponding dielectric functions $\epsilon(i \xi)$ display the largest and the least deviation from the handbook data (see Fig. 11 of Ref. 22]). Sample 4 on the other hand is the sample for which the Drude parameters were found to have the maximum uncertainties.

We evaluated the dielectric functions $\epsilon(i \xi)$ for these three samples, by using our windowed dispersion formula, and the traditional KK formula. In Fig. 14 we display the fractional difference $\left(\epsilon_{\mathrm{win}}(i \xi)-\epsilon_{\mathrm{KK}}(i \xi)\right) / \epsilon_{\mathrm{KK}}(i \xi)$ (in per cent) between the windowed and the KK estimates of the dielectric functions for these three samples, versus the imaginary frequency. When computing, by either method, the quantity $\epsilon_{\text {expt }}(i \xi)$ in Eq. (18), the handbook data were used for frequencies larger than $9 \mathrm{eV} / \hbar$. This is not expected to have much of an impact on the obtained results, because these large frequencies do not contribute much to $\epsilon(i \xi)$ in the considered range of imaginary frequencies. We observe first of all that the window estimates for $\epsilon(i \xi)$ corresponding to the two choices of the parameter $b$ agree within half per cent with each other. This fact proves the good degree of KK consistency of the optical data of [22]. Interestingly, however, the three samples display significant differences for what concerns agreement of the window and KK estimates. While for sample 5 the window estimates agree within less than one per cent with the KK values, for sample 1 and sample 4 significant deviations up to $6 \%$ and $10 \%$ respectively are found, the window approach providing systematically larger values than the KK method. By looking at the thick solid lines in Fig. 5 one can see that the obtained discrepancies are too large to be explained by uncertainties in the Drude parameters for these film, listed in TableII. We speculate that the found discrepancies between the windowed and the KK values obtained for samples 1 and 4 are related with deviations of the 


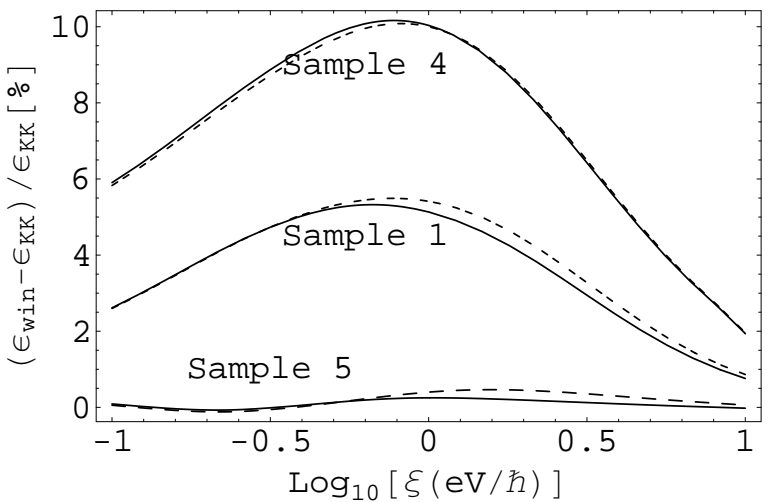

FIG. 14: Per cent difference between the KK and the window estimates for the permittivity $\epsilon(i \xi)$ for samples 1,4 and 5 of Ref. 22]. The window function is as in Eq. (13). Solid lines are for $b=1 \mathrm{eV} / \hbar$ and dashed lines for $b=1.5 \mathrm{eV} / \hbar$. The Drude parameters used to extrapolate the data are listed in Table II

dielectric function for these films from the Drude model, evidenced by the presence reported in 22] of unexplained absorption bands in the IR, for the samples $(1,2,3$ and 4) deposited on Si substrates (see Fig. 7 of [22]). The large errors in the Drude parameters that were obtained for sample 4 probably reflect the particular inadequacy of the Drude model for this sample. It is quite possible therefore that the KK estimate of $\epsilon(i \xi)$, which heavily relies on the Drude extrapolation (see Fig. 4), is much less accurate for samples 1 and 4 . In Fig. 15 we display the fractional difference $\left[P_{\mathrm{win}}(a, T)-P_{\mathrm{KK}}(a, T)\right] / P_{\mathrm{KK}}(a, T)$ (in per cent) between the windowed and the KK pressures versus plate separation (in microns) for samples 1, 4 and 5 . As we see the window and the standard KK predictions for the Casimir pressure agree within less than 0.1 per cent in the case of sample five, while for sample 1 and for sample 4 the window method predicts a stronger attraction than the standard KK approach. The theoretical uncertainty in the window prediction of the pressure, that can be estimated by considering the difference between the obtained pressures for the two considered values of the window parameter $b$, is less than 0.15 per cent for all samples and all displayed separations.

\section{PLASMA PRESCRIPTION}

In recent years it has been claimed that the Drude prescription leads to results that are in contradiction with precise measurements of the Casimir force in the submicron region [11]. However, the recent experiment [13] has been interpreted as being fully consistent with the Drude prescription. Independently of its accordance with experiments it has been claimed that the Drude prescription, when used at cryogenic temperatures in the idealized case of samples without defects, leads to a violation of the Nernst heat theorem [8]. For a detailed

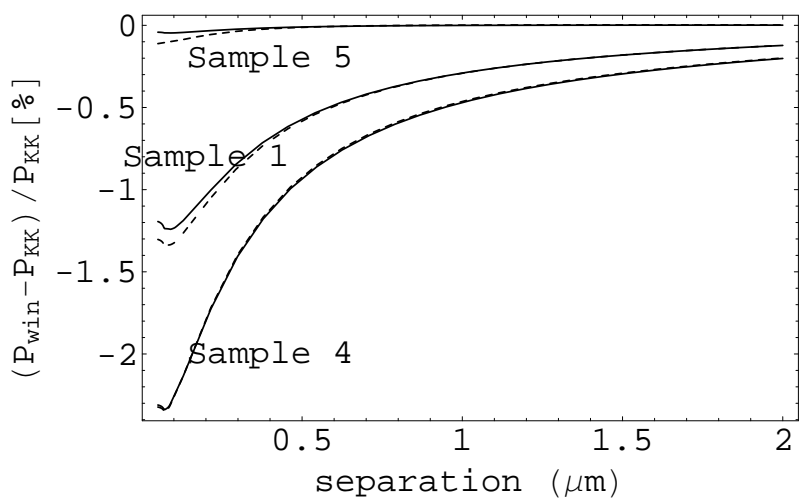

FIG. 15: Per cent difference between the window and the KK estimates for the Casimir pressure between two gold plates, for samples 1, 4 and 5 of Ref. 22]. The windowed pressure $P_{\text {win }}$ was computed using Eq. (13), with $b=1 \mathrm{eV} / \hbar$ (solid line) and $b=1.5 \mathrm{eV} / \hbar$ (dashed line). The Drude parameters used to extrapolate the data are listed in Table II

and updated discussion of these topics we address the reader to the monograph [2]. It has been argued that the above difficulties point to a fundamental flaw in the Drude approach, connected with relaxation processes of conduction electrons, and it has been suggested that the above deficiencies of the Drude prescription can be resolved if relaxation processes of conduction electrons are neglected when evaluating Lifshitz formula. According to this new scheme, the quantity $\epsilon(i \xi)$ to be plugged in Lifshitz formula should not be identified with the actual dielectric function of the metal, which includes such dissipative processes. One should rather use a modification of the dielectric function, so constructed as to disregard relaxation processes of conduction electrons. The modified expression of the permittivity, given in Eqs. (23) and (25) below, has been dubbed generalized plasma model, and following [2] we shall denote it by $\epsilon_{\mathrm{gp}}(i \xi)$. The name originates from the fact that within this model conduction electrons are described by the undamped plasma model $\epsilon_{p}(\omega)$ of infrared physics

$$
\epsilon_{p}(\omega)=1-\frac{\omega_{p}^{2}}{\omega^{2}}
$$

to which the Drude model Eq. (2) reduces when the dissipation parameter $\gamma$ is set to zero. We note that the generalized plasma model is not completely immune of troubles either, because its $1 / \omega^{2}$ singularity entails violation of the Bohr-van Leeuwen theorem of classical statistical physics, which is expected to hold for normal metals [10]. In Ref. [2] the following formula was obtained for $\epsilon_{\mathrm{gp}}(i \xi)$ :

$$
\epsilon_{\mathrm{gp}}(i \xi)=1+\frac{\omega_{p}^{2}}{\xi^{2}}+\frac{2}{\pi} \int_{0}^{\infty} \frac{d \omega \omega}{\omega^{2}+\xi^{2}} \epsilon_{\mathrm{core}}^{\prime \prime}(\omega),
$$

where the second term on the r.h.s. accounts for conduction electrons, now described as a collisionless plasma, 
and $\epsilon_{\text {core }}^{\prime \prime}(\omega)$ denotes the contribution of core electrons. The plasma frequency occurring in Eq. (23) must be extracted from the data. In the case of gold, the authors of 11] adopted the values $\omega_{p}=8.9 \mathrm{eV} / \hbar$ and $\gamma=0.0357$ $\mathrm{eV} / \hbar$, that were obtained from resistivity measurements performed at several different temperatures on their samples.

An equivalent representation of the generalized plasma dielectric function $\epsilon_{\mathrm{gp}}(i \xi)$, using the windowed dispersion relation Eq. (14), can be obtained by using the following representation for $\epsilon_{\text {core }}(i \xi)$ :

$$
\begin{aligned}
\epsilon_{\text {core }}(i \xi)= & \frac{2}{\pi} \sqrt{1+\left(\frac{b}{\xi}\right)^{2}}\left[-\int_{0}^{b} d \omega \frac{\omega^{2}}{\omega^{2}+\xi^{2}} \frac{\epsilon_{\text {core }}^{\prime}(\omega)}{\sqrt{b^{2}-\omega^{2}}}\right. \\
& \left.+\int_{b}^{\infty} d \omega \frac{\omega^{2}}{\omega^{2}+\xi^{2}} \frac{\epsilon_{\text {core }}^{\prime \prime}(\omega)}{\sqrt{\omega^{2}-b^{2}}}\right]
\end{aligned}
$$

The equivalent representation of $\epsilon_{\mathrm{gp}}(i \xi)$ then follows if we replace the second term on the r.h.s. of Eq. (23) representing the core contribution, by the above representation of $\epsilon_{\text {core }}(i \xi)$ :

$$
\begin{gathered}
\epsilon_{\mathrm{gp}}(i \xi)=1+\frac{\omega_{p}^{2}}{\xi^{2}}+\frac{2}{\pi} \sqrt{1+\left(\frac{b}{\xi}\right)^{2}}\left[-\int_{0}^{b} d \omega \frac{\omega^{2}}{\omega^{2}+\xi^{2}}\right. \\
\left.\times \frac{\epsilon_{\text {core }}^{\prime}(\omega)}{\sqrt{b^{2}-\omega^{2}}}+\int_{b}^{\infty} d \omega \frac{\omega^{2}}{\omega^{2}+\xi^{2}} \frac{\epsilon_{\text {core }}^{\prime \prime}(\omega)}{\sqrt{\omega^{2}-b^{2}}}\right] .
\end{gathered}
$$

If the frequency $b$ is chosen to be smaller than the onset frequency $\omega_{\text {inter }}$ of interband transition, the quantity $\epsilon_{\text {core }}^{\prime}(\omega)$ can be regraded as a constant $\bar{\epsilon}_{\text {core. }}$. The first integral on the r.h.s. of the above Equation can then be evaluated exactly. After doing it we obtain the following formula for $\epsilon_{\mathrm{gp}}(i \xi)$ :

$$
\begin{gathered}
\epsilon_{\mathrm{gp}}(i \xi)=1+\frac{\omega_{p}^{2}}{\xi^{2}}+\bar{\epsilon}_{\mathrm{core}}\left[1-\sqrt{1+\left(\frac{b}{\xi}\right)^{2}}\right] \\
+\frac{2}{\pi} \sqrt{1+\left(\frac{b}{\xi}\right)^{2}} \int_{b}^{\infty} d \omega \frac{\omega^{2}}{\omega^{2}+\xi^{2}} \frac{\epsilon_{\mathrm{core}}^{\prime \prime}(\omega)}{\sqrt{\omega^{2}-b^{2}}} .
\end{gathered}
$$

The above formula can be used as a substitute for Eq. (23) to compute $\epsilon_{\mathrm{gp}}(i \xi)$ starting from optical data.

\section{CONCLUSIONS AND DISCUSSION}

In recent years, much attention has been devoted to theoretical and experimental studies on the thermal Casimir effect between two metallic bodies. From the theory point of view the problem is that of understanding whether when computing the Casimir force, conduction electrons should be modelled as a collisionless plasma, as it is done in infrared physics, or whether ohmic relaxation processes should be fully considered. The two alternatives have been dubbed as plasma and Drude prescriptions, respectively. Depending on the chosen approach, different predictions result for the magnitude of the thermal Casimir force. The present experimental status of the problem is perplexing, because contradictory results have been obtained by different experiments. On one hand, a series of experiments [11] using a micro-torsional oscillator to measure the Casimir force in the short separation range from $160 \mathrm{~nm}$ to $750 \mathrm{~nm}$, were shown to be in agreement with the plasma prescription and to rule out the Drude prescription. Also a large distance torsion balance experiment [12] in which the Casimir force was measured in the separation range from 0.48 to $6.5 \mu \mathrm{m}$, obtained results that are in agreement with the assumption of ideal metal plates, and are in contradiction with the Drude model. On the contrary a new large distance torsional balance experiment [13], probing the Casimir force between a large spherical lens and a flat plate in the range from $700 \mathrm{~nm}$ to $7 \mu \mathrm{m}$, was claimed to be fully consistent with the Drude prescription, and to rule out the plasma model.

Motivated by the puzzling problem of the thermal Casimir effect, in the present paper we have developed mathematical tools that permit to obtain reliable predictions for the Casimir force in experiments using metallic plates, like those described above. This is an indispensable requisite for an assessment of the plasma versus Drude conundrum, when sub micron separations are considered. This is so because for separations smaller than half a micron the plasma and the Drude models predict magnitudes of the Casimir force that differ by just a few per cent, and therefore it is essential to make sure that the Casimir force can be actually predicted with per cent precision or better. Our work aims at resolving a difficulty that arises when one tries to obtain an accurate prediction for the Casimir force, starting from optical data for the plates of the Casimir apparatus. We considered specifically the case of gold films, which are used in the experiments described above. As it is well known, in order to compute the Casimir force, one needs to know the dielectric function $\epsilon(i \xi)$ of the involved materials, along the imaginary frequency axis. This function cannot be measured directly, and usually it is computed by means of a KK formula, which expresses $\epsilon(i \xi)$ in terms of optical data for the (imaginary part of the) measurable dielectric function $\epsilon(\omega)$. The difficulty addressed in this paper originates from the fact that optical data of gold are as a rule available only for wavelengths $\lambda$ shorter than a maximum wavelength belonging to the IR region. This is the case for example for the widely used handbook data in [20], where the complex index of refraction for gold is tabulated for wavelengths smaller than about 10 microns. The same is also true for the recent measurements of [22], where the dielectric function $\epsilon(\omega)$ for several gold films was measured by ellipsomtery in the range from 33 
to $0.14 \mu \mathrm{m}$. The problem is that knowing the dielectric function of gold for these wavelengths, one cannot obtain an accurate estimate of the KK integral for $\epsilon(i \xi)$. This so because the $1 / \omega$ singularity displayed by the dielectric function of ohmic conductors, makes low frequencies give a very large contribution to the the KK integral. Until now this difficulty has been resolved by extrapolating available optical data towards zero frequency by means of the simple Drude formula, which is known to provide a reasonable approximation to the dielectric function of ohmic conductors below the interband transition. Since the Drude extrapolation gives a very large contribution to the KK integral, the obtained predictions for the Casimir force are affected by a significant uncertainty, caused by experimental errors in the values of the Drude parameters. It was estimated in [22] that experimental uncertainties in the Drude parameters easily determine a one per cent uncertainty in the Casimir force between two gold plates at separations about $100 \mathrm{~nm}$. One cannot hope to resolve the problem by further extending optical measurements to lower frequencies, because for this purpose one would have to reach the Tera-Hertz region, which is practically very difficult.

Elaborating on an earlier proposal by the author 25], we have shown in this paper that it is possible to resolve this difficulty by using modified dispersion relations to evaluate $\epsilon(i \xi)$. The new form of dispersion relations involves certain weight functions, that we called window functions, that are aimed at suppressing the contribution of low frequencies, for which no optical data are available. The key feature of the new dispersion relations, as contrasted with the standard KK formula, is that they involve both the real and the imaginary parts of the permittivity. This does not constitute a problem, because refined optical techniques like ellipsometry permit to measure both quantities accurately [22]. The window functions introduced in our first work produced unsatisfactory results, when applied to the handbook data for gold [15]. In this paper, we demonstrated by means of a Monte Carlo simulation that the bad performance of these window functions originated from the fact that they lead to a large amplification of possible small experimental errors in the optical data. In this paper we have addressed this instability by introducing a new class of weight functions, which differently from the old ones lead to a positive definite kernel in the dispersion formula, in such a way that no instability occurs anymore. We have shown that the improved choice of window functions efficiently suppresses the contribution of low frequencies, in such a way that it is now possible to compute with high precision the Casimir pressure between two gold plates in vacuum, using currently available optical data, like the handbook data [20] and the new data of Ref. [22].

When we used the handbook data, we found it necessary to exclude from our computations all data for frequencies less than $0.6 \mathrm{eV} / \hbar$, whose original source was Ref. [29]. The reason for not using these IR data is that the film used by these authors appears to posses a plasma frequency of $7.7 \mathrm{eV} / \hbar$, which is exceedingly smaller than the value of $9.2 \mathrm{eV} / \hbar$ that we obtained from Theye's data [30], which are part of the handbook data from the near IR to the UV. The latter value of the plasma frequency is very close to the commonly accepted value of $9 \mathrm{eV} / \hbar$ [24]. After excluding the data of [29] the window method produces less accurate results, because the residual handbook data that ar left over start from a rather large frequency of $0.6 \mathrm{eV} / \hbar$. Nevertheless, the Casimir pressure can still be estimated by the window method with an uncertainty that we estimated smaller than 0.3 $\%$. When the Drude prescription is used, the values of the Casimir pressure obtained by the window method agree with those obtained by the standard approach, based on KK relations and the Drude extrapolation, within 0.2 $\%$ for separations larger than $50 \mathrm{~nm}$, and therefore they remain inconsistent with the experimental data of [11].

A much higher precision can be obtained when the data of 22] are used. Thanks to the fact that these data extend to longer wavelengths than the tabulated data, the uncertainty in the windowed prediction for the Casimir pressure decreases to $0.1 \%$, for separations larger than $50 \mathrm{~nm}$. This is an order of magnitude better than the $1 \%$ uncertainty that was estimated in 22] to result from uncertainty in the Drude parameters, within the standard KK approach. The magnitudes of the Casimir pressure predicted by the window method were found to be in very good agreement with predictions based on the standard KK formula for the mica sample of [22]. However for the two samples deposited on $\mathrm{Si}$ substrates that we considered significant discrepancies were found, the windowed Casimir pressures exceeding the KK values by more than $1 \%$ for sample 1 and by more than $2 \%$ for sample 4 , for separations less than $200 \mathrm{~nm}$. The discrepancy for samples 1 and 4 is too large to be explained by uncertainty in the Drude parameters, and we attribute it to the fact that the Drude model was shown in 22] not to describe well the dielectric function of these films in the IR, as evidenced by the presence in their optical data of an unexplained IR absorption band. It is quite possible that for this reason predictions for the Casimir force based on the Drude model are less accurate for these films.

In conclusion our results demonstrate that the window approach can be used to predict the Casimir pressure between two metallic plates very accurately, on the basis of standard optical data extending from the IR to the UV, without the need of further extending optical measurements to longer wavelengths than those reached by current laboratory optical apparatus. We hope that the results of this work will help clarifying present difficulties raised by the interpretation of experiments probing the thermal Casimir effect.

Acknowledgements The author thanks the ESF Research Network CASIMIR for financial support. Warm thanks are also due to G. L. Klimchitskaya and V. M. Mostepanenko for their constructive criticism, which greatly helped the author improving the manuscript, and to the authors of Ref. 22] for sharing their data. 
The author acknowledges also interesting correspondence with G. Palasantzas on the consequences of incomplete
Kramers-Kronig consistency of optical data.
[1] V. A. Parsegian, Van der Waals Forces (Cambridge University Press, Cambridge, England, 2005).

[2] M. Bordag, G. L.Klimchitskaya, U. Mohideen, and V. M. Mostepanenko, Advances in the Casimir Effect (Oxford University Press, Oxford, 2009).

[3] G. L. Klimchitskaya, U. Mohideen, and V. M. Mostepanenko, Rev. Mod. Phys. 81, 1827 (2009).

[4] S.K. Lamoreaux, Phys. Rev. Lett. 78, 5 (1997); U. Mohideen and A. Roy, ibid. 81, 4549 (1998); G. Bressi, G. Carugno, R. Onofrio and G. Ruoso, ibid. 88041804 (2002).

[5] M. Bostrom and B.E. Sernelius, Phys. Rev. Lett. 84, 4757 (2000); B.E. Sernelius, ibid. 87, 139102 (2001).

[6] G. Bimonte, New. J. Phys. 9, 281 (2007)

[7] F. Intravaia and C. Henkel, Phys. Rev. Lett. 103, 130405 (2009).

[8] V. B. Bezerra, G. L.Klimchitskaya, V. M. Mostepanenko, and C. Romero, Phys. Rev. A 69, 022119 (2004).

[9] I. Brevik, J.B. Aarseth, J.S. Hoye, and K.A. Milton, Phys. Rev. E 71, 056101 (2005.)

[10] G. Bimonte, Phys. Rev. A 79, 042107 (2009).

[11] R.S. Decca, D. Lopez, E. Fischbach, G.L. Klimchitskaya, D.E. Krause, and V.M. Mostepanenko, Ann. Phys. 318, 37 (2005); Phys. Rev. D 75, 077101 (2007); Eur. Phys. J. C 51, 963 (2007).

[12] M. Masuda and M. Sasaki, Phys. Rev. Lett. 102, 171101 (2009).

[13] A.O. Sushkov, W.J. Kim, D.A.R. Dalvit, and S.K. Lamoreaux, Nature Phys. 7, 230 (2011).

[14] V. B. Bezerra, G. L.Klimchitskaya, U. Mohideen, V. M. Mostepanenko, and C. Romero, Phys. Rev. B 83, 075417 (2011).

[15] B. Geyer, G. L. Klimchitskaya, and V. M. Mostepanenko, Phys. Rev. B81, 245421 (2010);

[16] B. Geyer, G. L. Klimchitskaya, and V. M. Mostepanenko, Phys. Rev. A82, 032513 (2010).
[17] H. B. Chan, V.A. Aksyuk, R.N. Kleiman, D.J. Bishop, and F. Capasso, Science 291, 1941 (2001).

[18] M. Lisanti, D. Iannuzzi, and F. Capasso, Proc. Nat. Acad. Sci. 102, 11989 (2005).

[19] S. de Man, K. Heeck, R. J. Wijngaarden, and D. Iannuzzi, Phys. Rev. Lett. 103, 040402 (2009).

[20] Handbook of Optical Constants of Solids, edited by E. D. Palik (Academic, New York, 1995).

[21] S. K. Lamoreaux, Phys. Rev. A 59, R3149 (1999).

[22] V. B. Svetovoy, P. J. van Zwol, G. Palasantzas, and J. Th. M. De Hosson, Plys. Rev. B 77, 035439 (2008).

[23] I. Pirozhenko, A. Lambrecht, and V. B. Svetovoy, New. J. Phys. 8, 238 (2006).

[24] A. Lambrecht and S. Reynaud, Eur. Phys. J. D 8, 309 (2000).

[25] G. Bimonte, Phys. Rev. A81, 062501 (2010).

[26] L. D. Landau, and E. M. Lifshitz, Landau and Lifshitz Course of Theoretical Physics: Electrodynamics of Continuous Media (Pergamon Press, New York, 1960).

[27] E. M. Lifshitz, Sov. Phys. JETP 2, 73 (1956); E. M. Lifshitz and L. P. Pitaevskii, Landau and Lifshitz Course of Theoretical Physics: Statistical Physics Part II (Butterworth-Heinemann, 1980).

[28] G. L. Klimchitskaya, U. Mohideen, and V. M. Mostepanenko, J. Phys. A Math. Theor. 40, 339(F) (2007).

[29] B. Dold and R. Mecke, Optik 22, 435 (1965).

[30] M. L. Theye, Phys. Rev. B 2, 3060 (1970).

[31] L. R. Canfield, G. Hass, and W. R. Hunter, J. Physique 25, 124 (1964).

[32] H. J. Hagemann, W. Gudat, and C. Kunz, J. Opt. Soc. Am. 65, 742 (1975).

[33] G. Grosso and G. Pastori Parravicini, Solid State Physics (Elsevier Academic Press, London, 2000).

[34] A. Lambrecht and S. Reynaud, Eur. Phys. J. D 8, 309 (2000). 